\title{
Computation of ESR spectra from the time evolution of the magnetization: Comparison of autocorrelation and Wiener-Khinchin-relation-based methods
}

\author{
Hiroki Ikeuchi, ${ }^{1,2, *}$ Hans De Raedt, ${ }^{3}$ Sylvain Bertaina, ${ }^{4}$ and Seiji Miyashita ${ }^{1,2}$ \\ ${ }^{1}$ Department of Physics, Graduate School of Science, The University of Tokyo, 7-3-1 Bunkyo-Ku, Tokyo, 113-0033, Japan \\ ${ }^{2}$ CREST, JST, 4-1-8 Honcho Kawaguchi, Saitama, 332-0012, Japan \\ ${ }^{3}$ Zernike Institute for Advanced Materials, University of Groningen, Nijenborgh 4, NL-9747AG Groningen, The Netherlands \\ ${ }^{4}$ Aix-Marseille Université, CNRS, IM2NP UMR7334, F-13397 Marseille Cedex 20, France
}

(Received 21 July 2015; revised manuscript received 18 October 2015; published 21 December 2015)

\begin{abstract}
The calculation of finite temperature electron spin resonance (ESR) spectra for concrete specified crystal configurations is a very important issue in the study of quantum spin systems. Although direct evaluation of the Kubo formula by means of numerical diagonalization yields exact results, memory and CPU time restrictions limit the applicability of this approach to small system sizes. Methods based on the time evolution of a single pure quantum state can be used to study larger systems. One such method exploits the property that the expectation value of the autocorrelation function obtained for a few samples of so-called thermal typical states yields a good estimate of the thermal equilibrium value. In this paper, we propose a new method based on a Wiener-Khinchin-like theorem for quantum system. By comparison with exact diagonalization results, it is shown that both methods yield correct results. As the Wiener-Khinchin-based method involves sampling over thermal typical states, we study the statistical properties of the sampling distribution. Effects due to finite observation time are investigated and found to differ for the two methods but it is also found that, for both methods, the effects vanish as the system size increases. We present ESR spectra of the one-dimensional XXZ Heisenberg chain of up to 28 spins and discuss the dependence of separation of double peaks on the chain length.
\end{abstract}

DOI: 10.1103/PhysRevB.92.214431

PACS number(s): 05.30.-d, 75.10.Jm, 76.30.-v

\section{INTRODUCTION}

Quantum spin systems have attracted interests for decades because they exhibit various nontrivial behavior due to quantum-mechanical effects. In particular, in low dimensions, quantum fluctuations due to noncommutativity of the spin operators and/or competition among the interaction (frustration) play an important role and various novel concepts, such as the valence-bond solid, resonating-valence bonds, and magnon Bose-Einstein condensation, etc., have been developed. One important topic is the effect of nonmagnetic defects. In a spin $S=1 / 2$ antiferromagnetic Heisenberg chain, quantum fluctuations prevent the spins from being ordered, even at $T=0 \mathrm{~K}$, and the ground state is nonmagnetic. However, nonmagnetic defects break the translational symmetry and polarize the surrounding spins [1-3]. Then the one-dimensional system is described by an open-ended spin chain. An example of such a system is the $\mathrm{Pd}$ doped chain $\mathrm{Sr}_{2} \mathrm{CuO}_{3}$ [4-6]. As to the dimerized system, recently correlated defects in an alternate chain (TMTTF) ${ }_{2} \mathrm{PF}_{6}$ have been observed [7]. While in the latter system, the electron spin resonance (ESR) spectrum has been found thanks to its spin structure with defects, in the former case the system is uniform and the edge effect spreads widely, and thus only susceptibility has been observed, but no ESR has been reported yet. In this way, it has attracted interests that impurity or spatially inhomogeneity induce a magnetization moment in a nonmagnetic singlet state. To study properties of ESR of such magnetization, we need to treat a large-enough lattice which can include a profile of magnetic moments which are extended over a number of lattice sites. Thus, it is desirable to develop method to study larger lattices.

*Corresponding author: ikeuchi@spin.phys.s.u-tokyo.ac.jp
ESR is one of the major tools to study the effects of defects in spin systems. Modeling the ESR spectra of intrinsic defects in spin chains is an important problem for which data for finite but rather long chains are necessary. In particular, the parameter dependence of concrete ESR spectrum for a specified system is of great interest and the temperature dependence of the ESR spectrum provides a lot of information about the spin ordering. To study these aspects theoretically, the explicit form of interactions and spatial configuration of magnetic ions in the lattice play an important role and it is necessary to study microscopic models, that is we should calculate the ESR spectrum for specific quantum spin Hamiltonian. The most direct way is to calculate the Kubo formula [8,9] by making use of the eigenvalues and their eigenvectors obtained by diagonalization of the Hamiltonian. The first attempt has been made to study the Nagata-Tazuke phenomena [10] in a one-dimensional Heisenberg chain of eight spins with dipole-dipole interactions [11]. In this work, the dependence of the spectrum on the angle between the static field and lattice direction was reproduced. Moreover, antiferromagnetic resonance [12] and also the appearance of a resonance forbidden by the Dzyaloshinskii-Moriya interaction [13] were studied. The structure of the ESR spectrum of a spin ring at high temperatures was investigated up to 16 spins [14]. In complementary work, Oshikawa and Affleck have developed a field theoretical approach by making use of the exact autocorrelation of Heisenberg chain and have successfully analyzed low temperature properties for long (infinite) chains [15].

Obviously, the application of this method of exact diagonalization (ED) is limited to small systems for which we can obtain all the eigenvalues and their eigenvectors. For a system of $N S=1 / 2$ spins, we need the memory of order $2^{2 N}$. By making use of the symmetries of the system we may reduce 
the dimensions of the block diagonalized Hamiltonian. Even with these efforts, in practice, the system size is still limited to about 20 spins.

The restriction imposed by the exact diagonalization approach can be alleviated by computing the autocorrelation function (AC) from the time evolution of a pure state [16-18]. The ESR spectrum is obtained by Fourier transform of the autocorrelation function of the transverse magnetization. In the AC method, a pure state evolves in time according to the time-evolution operator $e^{-i \mathscr{H} t}$ the action of which can be computed efficiently by means the Chebyshev polynomial expansion [19-22] or the Suzuki-Trotter-product-formula method [16,23]. As the memory required to store one pure state or all eigenstates is of the order $2^{N}$ and $2^{2 N}$, respectively, the time evolution method allows us to study systems that are twice the size of those that can be studied by exact diagonalization. For compute thermal averages we need, in principle, to take the average over the initial states. However, it is known that the expectation value of a quantity $A$ for a single random state $|\Phi\rangle$ yields an estimate of the trace of $A$, that is $\langle\Phi|A| \Phi\rangle \simeq \operatorname{Tr} A$, which becomes more accurate as the size of Hilbert space increases [23]. Because of this fact, there is no need to compute traces of matrices to obtain the thermal equilibrium average [17,18,23-25]. This approach has been used to study the temperature dependence of the total amplitude of ESR spectrum for the single molecular magnet $\mathrm{V}_{15}$, consisting of $15 S=1 / 2$ spins [18]. Although this method to compute the ESR spectrum has considerable potential, properties of its applications to large systems have not yet been scrutinized in sufficient detail.

An important issue, which we address in this paper, is that because the time-evolution method necessarily yields data for a finite time interval only, it is important to study how the ESR spectrum, that is, the Fourier transform of this data, depends on the size of the time interval.

An early application of the time-domain method to spin- $1 / 2$ models reported results for the density of states and, e.g., the dynamic structure factor of systems up to 26 spins [16]. At present, it is possible to study, e.g., thermalization/decoherence [26] and compute time-dependent transport properties [27] for systems containing 34 and 36 spins, respectively. In the present paper, we study how we can obtain ESR spectrum by making use of the time-domain method. Although calculation of the ESR spectrum amounts to calculating a specific transport property (see below), the nature of the physics involved puts some constraints on the system sizes that can be studied. The main problem here is that to compare with actual ESR experiments, it is necessary to be able to resolve detailed structures in the spectra. This requires knowledge of the spin dynamics over a (very) large time interval (see below), much larger than in the case of the dynamic structure factor [16] or spin diffusion [27], for instance. Furthermore, the fact that the interval is finite causes various artifacts. Thus, at present, the finite observation time and artifacts due to the finite sampling, not the number of spins, is the dominant limiting factor in the calculation of the ESR spectrum.

The effect of finite observation time in AC method is serous in small systems which were used to test. If we apply usual gauss window method, then the fine structure of the spectrum is smeared out, and thus we thought the method is not good practically, and it has not been used practical calculation in spin systems.

But in the present paper, we clarified the nature of the effect of finite observation time. In the $\mathrm{AC}$ method, the spectrum is obtained from the autocorrelation function $\left\langle M^{x} M^{x}(t)\right\rangle_{\mathrm{eq}}$. In ESR experiments, one measures the time evolution of the magnetization $M^{x}(t)$. The relation between the $\mathrm{AC}$ and the spectral density $M^{x}(t)$ is given by the Wiener-Khinchin (WK) theorem. This theorem relates the spectral density of the dynamics of a quantity and the Fourier transform of the autocorrelation function of the same quantity. In the present paper, we propose a method to directly compute the ESR spectrum from the time evolution of $M^{x}(t)$ by exploiting the idea of WK theorem. I found that the effect of the finite observation time is significantly suppressed in the WK method. In particular, the Gibbs oscillations in the WK method are only positive, whereas they can be negative in the AC method. The WK method provides supplemental information about effects of finiteness of time domain.

In quantum systems, however, the definition of the magnetization dynamics is somewhat tricky, and we therefore develop a quantum version of Wiener-Khinchin relation, i.e., an explicit relation between Fourier transform of the autocorrelation function and the spectrum density in the quantum case. We call the approach based of this idea the WK method. Because the thermal average of the magnetization in the transverse direction $\left\langle M^{x}(t)\right\rangle_{\text {eq }}$ is zero, and the ESR signal is proportional to the average of square of the Fourier transform of $\left\langle\Phi\left|M^{x}(t)\right| \Phi\right\rangle$ over many realizations of the random state $|\Phi\rangle$, the reasoning about the convergence of this average as a function of the dimension of the Hilbert space does not hold. Therefore, we present a detailed analysis of the distribution of the sampled data and show that the distribution converges with a finite variance, independent of the size of Hilbert space. Because of the latter property, also for a large system, it is necessary to perform ensemble averaging of the data which renders the computational efficiency of the WK method less than the one of the AC method.

As a fortunate property, we found that the effect of the finite observation time is suppressed even in the AC method when the size of the system becomes large. We confirmed this property in the system of $N=16$, for which we can obtain spectra by the $\mathrm{ED}, \mathrm{WK}$, and AC methods, and results of the methods without any window-smearing technique (e.g., Gauss window) give the same results. Because the AC method has better statistical property, i.e., because a single sample of a random state yields accurate result, once one knows that the effect of the finite observation time is suppressed in large systems, the AC method is the most efficient for large systems. We performed the $\mathrm{AC}$ method to the one-dimensional XXZ model which was studied previously and gives a good reference. We reproduced the double peak structure in the spectrum which was found in earlier work [14]. In the paper, whether the double-peak structure would disappear in longer chains has been discussed in the viewpoint of relaxation of the autocorrelation function of the force for the spin dynamics [14]. We present the separation of the double peak up to $N=28$. The separation is the same for $N=22,24$, and 26, but we find that it decreases again at $N=28$. Overall behavior may show decrease proportionally to the inverse of chain length, but it may show a tendency to 
saturate, and thus up to the present size we cannot make a conclusion on this property.

The outline of this paper is as follows. A brief overview on the methods previously used is given in Sec II. In Sec. III, we introduce the new method motivated by the Wiener-Khinchin theorem and study statistical properties of the method in detail in Sec. III C. In Sec. IV, we show the distribution of sampled data of the spectrum obtained by the AC and WK methods. The total amplitude of the spectrum, which is fundamental information in ESR studies, is discussed in Sec. V. Section VI presents applications of the methods to large systems. A summary and discussion of related problems are given in Sec. VII. Appendix A discusses the effect of the finite-time interval on the spectrum for both methods. We give the statistical properties of the thermal typical state and the detailed calculations of the conservation of total intensity in the Supplemental Materials in Refs. [28,29], respectively.

\section{RECAPITULATION OF EXISTING METHODS}

\section{A. Kubo formula and ESR spectrum}

The ESR spectrum is given by the Kubo formula [8,9]. The imaginary part of the dynamical susceptibility $\chi^{\prime \prime}(\omega)$ reads ${ }^{1}$

$$
\chi^{\prime \prime}(\omega)=\frac{1}{2}\left(1-e^{-\beta \omega}\right) \int_{-\infty}^{\infty}\left\langle M^{x}(0) M^{x}(t)\right\rangle_{\mathrm{eq}} e^{-i \omega t} d t,
$$

and the ESR absorption spectrum is given by

$$
I^{x}(\omega)=\frac{\omega \lambda_{0}^{2}}{2} \chi^{\prime \prime}(\omega)
$$

where $\lambda_{0}$ is the amplitude of the external field, and we adopt

$$
\begin{gathered}
M^{x}(t)=e^{i \mathscr{H} t} M^{x} e^{-i \mathscr{H} t}, \quad M^{x}=\sum_{i=1}^{N} S_{i}^{x}, \\
\langle\cdot\rangle_{\text {eq }}=\operatorname{Tr}\left[\cdot e^{-\beta \mathscr{H}}\right] / \operatorname{Tr}\left[e^{-\beta \mathscr{H}}\right] .
\end{gathered}
$$

For the numerical calculation of the ESR absorption spectrum, several methods have been developed. There are essentially two types of methods: (1) the exact diagonalization method $[11,14]$ and (2) the time evolution of the autocorrelation function method [17].

\section{B. Exact diagonalization (ED) method}

The most direct calculation of the Kubo formula uses the set of eigenvalues and eigenvectors $\left\{E_{n},|n\rangle\right\}_{n=1}^{D}$ obtained by solving the eigenvalue problem

$$
\mathscr{H}|n\rangle=E_{n}|n\rangle
$$

for the Hamiltonian $\mathscr{H}, D$ denoting the dimension of the Hilbert space. In practice, we solve Eq. (5) by numerical diagonalization. The autocorrelation function $\left\langle M^{x}(0) M^{x}(t)\right\rangle_{\mathrm{eq}}$ is expressed as

$$
\left\langle M^{x}(0) M^{x}(t)\right\rangle_{\mathrm{eq}}=\sum_{m, n}\left|\left\langle m\left|M^{x}\right| n\right\rangle\right|^{2} e^{i\left(E_{m}-E_{n}\right) t-\beta E_{n}} / Z,
$$

\footnotetext{
${ }^{1}$ Throughout the paper we denote the temperature by $\beta^{-1}$ in order to avoid confusion with the end of the time interval $T$.
}

where $Z$ is the partition function

$$
Z=\sum_{n} e^{-\beta E_{n}}
$$

The Fourier transform of Eq. (6) reads

$$
\begin{aligned}
& \int_{-\infty}^{\infty}\left\langle M^{x}(0) M^{x}(t)\right\rangle_{\mathrm{eq}} e^{-i \omega t} d t \\
& =\sum_{m, n}\left|\left\langle m\left|M^{x}\right| n\right\rangle\right|^{2} e^{-\beta E_{n}} 2 \pi \delta\left[\omega-\left(E_{m}-E_{n}\right)\right] / Z,
\end{aligned}
$$

where we used the definition

$$
\delta\left[\omega-\left(E_{m}-E_{n}\right)\right]=\frac{1}{2 \pi} \int_{-\infty}^{\infty} e^{-i\left[\omega-\left(E_{m}-E_{n}\right)\right] t} d t .
$$

The imaginary part of the dynamical susceptibility reads

$$
\chi^{\prime \prime}(\omega) \equiv \sum_{m, n} D_{m, n} \delta\left(\omega-\omega_{m, n}\right)
$$

where

$$
\begin{aligned}
D_{m, n} & \equiv \pi\left(e^{-\beta E_{n}}-e^{-\beta E_{m}}\right)\left|\left\langle m\left|M^{x}\right| n\right\rangle\right|^{2} / Z, \\
\omega_{m, n} & \equiv E_{m}-E_{n} .
\end{aligned}
$$

It is sufficient to consider $\omega_{m, n}>0$ only since we are interested in the absorption, not the emission. Note that $\chi^{\prime \prime}(\omega)>0$ for $\omega>0$.

In the exact diagonalization approach, the spectrum consists of a finite sum of $\delta$ functions. Therefore, to draw the spectrum, we have to represent each $\delta$ function by a regular function of certain width. For example, we may replace the $\delta$ function by a Gaussian or simply use a histogram representation. The results of the exact diagonalization are exact, usually close to machine precision, but we need to know all the eigenvalues and corresponding eigenstates. Therefore, we need memory of the order of $D^{2}$ for a matrix of the size $D=2^{N}$ for systems of $N S=1 / 2$ spins. If the system has symmetry, then we may reduce the size. For example, if the system conserves $M^{z}$, then $D$ is reduced to ${ }_{N} C_{N / 2+M^{z}}$. Moreover for ESR spectra, only the uniform mode is relevant and therefore only the fully symmetrized states are necessary, which also allows a reduction of $D$. However, the memory limitation prevents us from studying more than $N=20 S=1 / 2$ spins. As an illustration, we use the exact diagonalization approach to study the one-dimensional spin-1/2 XXZ model in a static magnetic field $H$ and compute the response of the magnetization along the $x$ axis to the $\mathrm{AC}$ field $\lambda(t)$. The Hamiltonian of the system is given by

$$
\begin{aligned}
\mathscr{H}_{\mathrm{tot}}= & \sum_{i=1}^{N}\left(J_{x} S_{i}^{x} S_{i+1}^{x}+J_{y} S_{i}^{y} S_{i+1}^{y}+J_{z} S_{i}^{z} S_{i+1}^{z}\right) \\
& -g \mu_{B} H \sum_{i=1}^{N} S_{i}^{z}+\lambda_{0} \cos \omega t \sum_{i=1}^{N} S_{i}^{x},
\end{aligned}
$$

where we impose the periodic boundary condition $S_{N+1}^{\alpha}=S_{1}^{\alpha}, \alpha=x, y, z$ unless otherwise mentioned. Hereafter we adopt Kelvin as the unit of energy and we set $g \mu_{B}=1$.

In Fig. 1, we show an example of spectrum obtained by exact diagonalization method for an antiferromagnetic Heisenberg chain with $N=16$ with the static field $H=5 \mathrm{~K}$ at the temperature $\beta^{-1}=100 \mathrm{~K}$. The notation and the parameters 


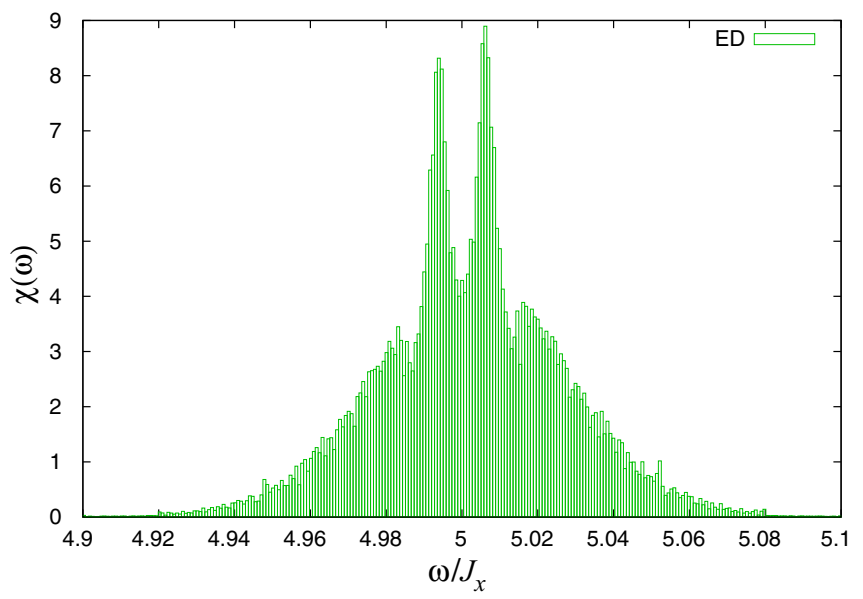

FIG. 1. (Color online) Spectrum for a ring of $N=16$ spins. The histogram is obtained by the exact diagonalization method.

of the model are set to be the same as those in the model studied in the previous study [14] $\left(J_{x}=J_{y}=1 \mathrm{~K}, J_{z}=0.92 \mathrm{~K}\right)$.

The spectrum consisting of an ensemble of the $\delta$ peaks [Eq. (10)] is plotted as a histogram with small bins $\Delta \omega$. In the exact diagonalization (ED) method, we are free to choose any value of $\Delta \omega$. Anticipating for the comparison with the two other methods, we take $\Delta \omega=2 \pi / T$, where $T=d t \times n_{t}$, $d t=0.5$, and $n_{t}=16384$ is the number of data points.

\section{Autocorrelation function method}

According to the Kubo formula [Eq. (1)], the spectrum is given by Fourier transform of the autocorrelation function. Because of the symmetry

$$
\left(\left\langle M^{x} M^{x}(t)\right\rangle_{\mathrm{eq}}\right)^{*}=\left\langle M^{x} M^{x}(-t)\right\rangle_{\mathrm{eq}},
$$

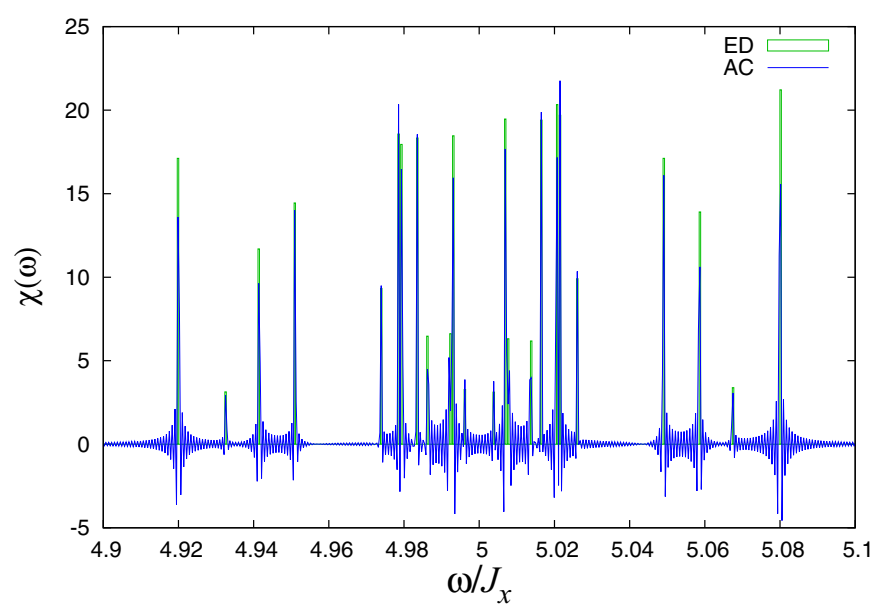

where $*$ denotes complex conjugate, we have

$$
\int_{-\infty}^{\infty}\left\langle M^{x} M^{x}(t)\right\rangle_{\mathrm{eq}} e^{-i \omega t} d t=2 \operatorname{Re}\left[\int_{0}^{\infty}\left\langle M^{x} M^{x}(t)\right\rangle_{\mathrm{eq}} e^{-i \omega t} d t\right],
$$

where $\operatorname{Re}[\cdot]$ denotes the real part. Therefore, to compute Eq. (14), it suffices to have AC data for the interval $[0, T]$.

We obtain the spectrum by discrete Fourier transform (DFT) of the AC data

$$
\begin{aligned}
f\left(t_{m}\right) & \equiv\left\langle M^{x} M^{x}\left(t_{m}\right)\right\rangle_{\mathrm{eq}}, \quad t_{m}=\frac{m T}{n_{t}}, \\
m & =0,1,2, \ldots, n_{t}-1,
\end{aligned}
$$

where $n_{t}$ is the number of data items. The DFT of Eq. (14) is given by

$$
\begin{aligned}
& \frac{T}{n_{t}} \sum_{m=-n_{t}}^{n_{t}-1} f\left(\frac{m T}{n_{t}}\right) e^{-i \omega_{k} m T / n_{t}}, \quad \omega_{k} \equiv \frac{\pi k}{T}, \\
& k=-n_{t},-n_{t}+1, \ldots, 0,1,2, \ldots, n_{t}-1 .
\end{aligned}
$$

Note that the absorption and emission spectrum correspond to $\omega_{k}>0$ and $\omega_{k}<0$, respectively. The maximum angular frequency ( $2 \pi$ times Nyquist frequency) that can be represented by the DFT Eq. (16) is given by $\pi n_{t} / T$. For a magnetic field $H=5 \mathrm{~K}$, the main contribution to the absorption spectrum peak comes from a narrow peak around $\omega \approx 5 \mathrm{~K}$. In our numerical work we take $d t \equiv T / n_{t}=0.5$. Therefore the largest value of omega ( $2 \pi$ times the Nyquist frequency) is $(2 \pi / T) \times\left(n_{t} / 2\right)=\pi / d t=2 \pi \approx 6.28>5$, which is large enough to cover the full spectral range.

In Fig. 2 (left), we show the spectrum for a system of $N=6$ spins at the temperature $\beta^{-1}=100 \mathrm{~K}$, obtained by using a time series of $n_{t}=16384$ items. Clearly, the spectrum is suffering from fine oscillations with negative values, which is called the Gibbs oscillation [30]. This oscillation is due to the fact that the range of the time integral is finite and that

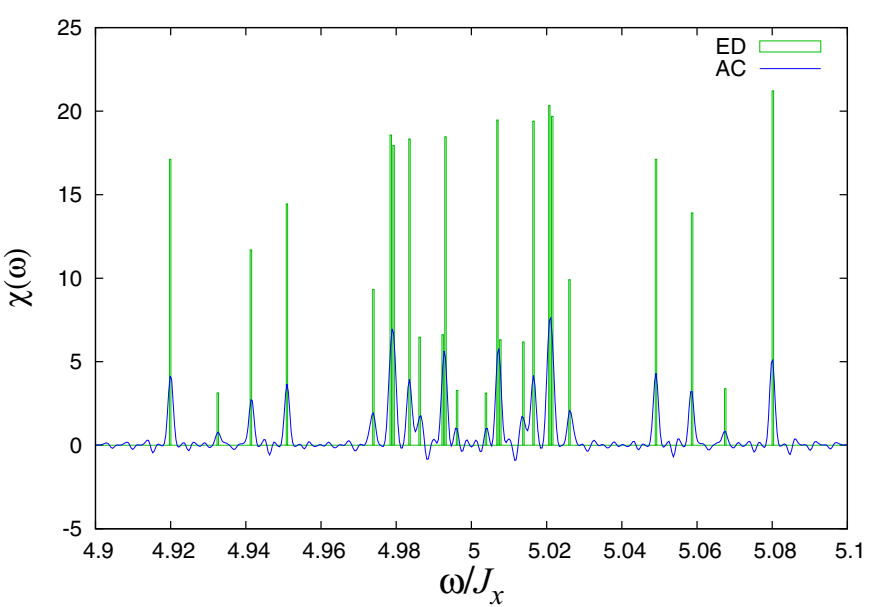

FIG. 2. (Color online) The spectrum obtained by the exact diagonalization method and the time-evolution method: $N=6$, temperature $\beta^{-1}=100 \mathrm{~K}, H=5 \mathrm{~K}$, sampling time $d t=0.5$, the number of data items $n_{t}=16384$, and the $\omega$ mesh $\pi / T=\pi /\left(n_{t} \times \mathrm{d} t\right)($ blue line). The exact diagonalization result (green line) uses an $\omega$ mesh $\Delta \omega=2 \pi / 16384$, which corresponds to the frequency $\pi / T$ used in the time-evolution method. Left: The spectrum obtained by exact diagonalization (green line) and by use of the time-evolution method without window functions (blue line). Strong Gibbs oscillation is found. Right: Same as left except that a Gaussian window function with standard deviation $\alpha / T=7 / 8192=0.00085(\alpha=7)$ is used. Clearly, the Gibbs oscillation is suppressed. 
there are eigenvalues that do not exactly match one of the $\omega_{k}$. This artifact can be suppressed by employing a suitable window function [30], for instance, a Gaussian window (see Appendix A). As shown in Fig. 2 (right), the Gibbs oscillation is reduced and the result is consistent with the one derived with the exact diagonalization method with the resolution of $O(\alpha)$ [ $\alpha$ is an artificial parameter which determines the resolution of the spectrum. See Eq. (A12).]

\section{Thermal typical state method}

In theory, the calculation of the thermal equilibrium expectation value of $M^{x} M^{x}(t)$ involves a trace operation. This requires of the order $D^{2}=2^{2 N}$ operations, which becomes prohibitively large if $N$ is of the order of 20 or larger. Fortunately, for larger systems, we can obtain accurate estimates of thermal equilibrium averages by making use of the so-called thermal typical state $\left|\Phi_{\beta}\right\rangle=e^{-\beta \mathscr{H} / 2}|\Phi\rangle[17,18,23,25]$, also called "a Boltzmann-weighted random vector" $[17,18]$ or "a canonical thermal pure quantum state" [25], where $|\Phi\rangle$ denotes a random state on the $D$-dimensional hypershere. In essence, we have [23]

$$
\langle X\rangle_{\mathrm{eq}}=\frac{\operatorname{Tr} X e^{-\beta \mathscr{H}}}{\operatorname{Tr} e^{-\beta \mathscr{H}}} \simeq\left\langle\Phi_{\beta}|X| \Phi_{\beta}\right\rangle .
$$

We briefly explain the ideas behind this method. Let $\{|n\rangle\}_{n=1}^{D}$ be an arbitrary set of complete orthonormal states of the Hilbert space of the system. Using the complex-valued random variables $\left\{\xi_{n}\right\}_{n=1}^{D}$, we introduce a random vector:

$$
|\Phi\rangle=\sum_{n=1}^{D} \xi_{n}|n\rangle
$$

Note that this construction is independent of the choice of the basis set $\{|n\rangle\}_{n=1}^{D}$.

Unlike in A. Hams and H. De Raedt [Phys. Rev. E 62, 4365 (2000)], to simplify the mathematics, we will work with independent complex-valued Gaussian random variables for each index $n$. They have identical Gaussian distributions with mean zero and variance $\sigma^{2}$ for all real and imaginary parts of the variables. By $E[\cdot]$, we denote the expectation with respect to the multivariate Gaussian probability distribution:

$$
\begin{aligned}
& P\left(\xi_{1}, \ldots, \xi_{D}\right) \prod_{a=1}^{D} d\left(\operatorname{Re} \xi_{a}\right) d\left(\operatorname{Im} \xi_{a}\right) \\
& \quad=\prod_{a=1}^{D}\left[\frac{1}{2 \pi \sigma^{2}} e^{-\left|\xi_{a}\right|^{2} / 2 \sigma^{2}}\right] d\left(\operatorname{Re} \xi_{a}\right) d\left(\operatorname{Im} \xi_{a}\right) .
\end{aligned}
$$

The following relations hold:

$$
\begin{aligned}
E\left[\xi_{a}\right] & =E\left[\xi_{p}\right]=E\left[\xi_{a} \xi_{b}\right]=0, \\
E\left[\xi_{a}^{*} \xi_{p}\right] & =2 \sigma^{2} \delta_{a, p}, \\
E\left[\xi_{a}^{*} \xi_{b}^{*} \xi_{p} \xi_{q}\right] & =E\left[\xi_{a}^{*} \xi_{p}\right] E\left[\xi_{b}^{*} \xi_{q}\right]+E\left[\xi_{a}^{*} \xi_{q}\right] E\left[\xi_{b}^{*} \xi_{p}\right] \\
& =4 \sigma^{4}\left(\delta_{a, p} \delta_{b, q}+\delta_{a, q} \delta_{b, p}\right),
\end{aligned}
$$

which are used in the following calculations.
For any matrix $X$ we have

$$
\begin{aligned}
E[\langle\Phi|X| \Phi\rangle] & =\sum_{a, p=1}^{D} E\left[\xi_{a}^{*} \xi_{p}\right]\langle a|X| p\rangle \\
& =\sum_{a}^{D} E\left[\xi_{a}^{*} \xi_{a}\right]\langle a|X| a\rangle=2 \sigma^{2} \operatorname{Tr} X
\end{aligned}
$$

and because $\left\langle\Phi\left|X^{\dagger}\right| \Phi\right\rangle=\langle\Phi|X| \Phi\rangle^{*}$, the corresponding variance is given by

$$
\begin{aligned}
\operatorname{Var} & (\langle\Phi|X| \Phi\rangle) \\
= & E\left[|\langle\Phi|X| \Phi\rangle|^{2}\right]-|E[\langle\Phi|X| \Phi\rangle]|^{2} \\
= & \sum_{a, p, b, q=1}^{D} E\left[\xi_{a}^{*} \xi_{p} \xi_{b} \xi_{q}^{*}\right]\langle a|X| p\rangle\langle b|X| q\rangle^{*}-4 \sigma^{4}|\operatorname{Tr} X|^{2} \\
= & 4 \sigma^{4} \sum_{a, b=1}^{D}\left(\langle a|X| a\rangle\langle b|X| b\rangle^{*}+\langle a|X| b\rangle\langle a|X| b\rangle^{*}\right) \\
& -4 \sigma^{4}|\operatorname{Tr} X|^{2} \\
= & 4 \sigma^{4} \operatorname{Tr} X X^{\dagger} .
\end{aligned}
$$

The relative standard deviation is defined by

$$
\begin{aligned}
\operatorname{RSD}^{2}(\langle\Phi|X| \Phi\rangle) & =\frac{\operatorname{Var}(\langle\Phi|X| \Phi\rangle)}{|E[\langle\Phi|X| \Phi\rangle]|^{2}}=\frac{\operatorname{Tr} X X^{\dagger}}{|\operatorname{Tr} X|^{2}} \\
& =\frac{\operatorname{Tr} X X^{\dagger}}{(\operatorname{Tr} X)\left(\operatorname{Tr} X^{\dagger}\right)} .
\end{aligned}
$$

Next, we introduce the thermal typical state $\left|\Phi_{\beta}\right\rangle$ for the system at an inverse temperature $\beta$ :

$$
\left|\Phi_{\beta}\right\rangle=e^{-\beta \mathscr{H} / 2}|\Phi\rangle,
$$

where $\left\langle\Phi_{\beta} \mid \Phi_{\beta}\right\rangle=\left\langle\Phi\left|\mathrm{e}^{-\beta \mathscr{H}}\right| \Phi\right\rangle$ is an approximation to the partition function $Z_{\beta}$. The RSD of $\left\langle\Phi\left|\mathrm{e}^{-\beta \mathscr{H}}\right| \Phi\right\rangle$ vanishes with $D$ as (see the Supplemental Material in Ref. [28])

$$
\operatorname{RSD}\left(\left\langle\Phi_{\beta} \mid \Phi_{\beta}\right\rangle\right)=\operatorname{RSD}\left(\left\langle\Phi\left|e^{-\beta \mathscr{H}}\right| \Phi\right\rangle\right) \lesssim \mathscr{O}\left(D^{-1 / 2}\right) .
$$

Thus, hereafter we only estimate the RSD of the numerator for the thermal average. In our calculations, we take averages of the dominator and the numerator with respect to the same samples $\left\{\left|\Phi_{\beta}\right\rangle\right\}$ :

$$
\langle X\rangle_{\mathrm{eq}} \simeq \frac{\frac{1}{N} \sum_{\left|\Phi_{\beta}\right\rangle}\left\langle\Phi_{\beta}|X| \Phi_{\beta}\right\rangle}{\frac{1}{N} \sum_{\left|\Phi_{\beta}\right\rangle}\left\langle\Phi_{\beta} \mid \Phi_{\beta}\right\rangle},
$$

where $N$ is the number of samples. But if we take the average of the ratios of samples, we obtain the same result in the limit $D \rightarrow \infty$ (see the Supplemental Material in Ref. [28]):

$$
\langle X\rangle_{\mathrm{eq}} \simeq \frac{1}{N} \sum_{\left|\Phi_{\beta}\right\rangle} \frac{\left\langle\Phi_{\beta}|X| \Phi_{\beta}\right\rangle}{\left\langle\Phi_{\beta} \mid \Phi_{\beta}\right\rangle}
$$

The autocorrelation function $\left\langle M^{x} M^{x}(t)\right\rangle_{\mathrm{eq}}$ is given by

$$
\left\langle M^{x} M^{x}(t)\right\rangle_{\mathrm{eq}}=\frac{E\left[\left\langle\Phi_{\beta}\left|M^{x} e^{i \mathscr{H} t} M^{x} e^{-i \mathscr{H} t}\right| \Phi_{\beta}\right\rangle\right]}{E\left[\left\langle\Phi_{\beta} \mid \Phi_{\beta}\right\rangle\right]} .
$$

The RSD of the autocorrelation function also vanishes with increasing $D$ (see the Supplemental Material in Ref. [28]). 
Thus, for a sufficiently large system, a few samples suffice to estimate the autocorrelation function. Here it should be noted that, strictly speaking, Eq. (13) does not hold for the quantity $\left\langle\Phi_{\beta}\left|M^{x} e^{i \mathscr{H} t} M^{x} e^{-i \mathscr{H} t}\right| \Phi_{\beta}\right\rangle$, but, for sufficiently large $D$, Eq. (28) justifies using Eq. (13).

For the autocorrelation function $\left\langle M^{x} M^{x}(t)\right\rangle_{\mathrm{eq}}$, we need to construct the states

$$
|A\rangle \equiv e^{-i \mathscr{H} t}\left|\Phi_{\beta}\right\rangle, \quad \text { and } \quad|B\rangle \equiv e^{-i \mathscr{H} t} M^{x}\left|\Phi_{\beta}\right\rangle,
$$

and then obtain the expectation value of the autocorrelation as

$$
\left\langle\Phi_{\beta}\left|M^{x} e^{i \mathscr{H} t} M^{x} e^{-i \mathscr{H} t}\right| \Phi_{\beta}\right\rangle=\left\langle B\left|M^{x}\right| A\right\rangle .
$$

To perform the operations $e^{-\beta \mathscr{H} / 2}$ and $e^{-i \mathscr{H} t}$ on the states, we may use the Chebyshev polynomial expansion method or the Suzuki-Trotter decomposition method. In the present study we used the Chebyshev method for $e^{-\beta \mathscr{H} / 2}|\Phi\rangle$ and mostly used the Suzuki-Trotter decomposition method for $e^{-i \mathscr{H} t}\left|\Phi_{\beta}\right\rangle$.

\section{WIENER-KHINCHIN METHOD}

In this section, we propose a new method that makes use of the Wiener-Khinchin theorem. In the autocorrelation method (see previous section) the spectrum is estimated from the autocorrelation function $\left\langle M^{x} M^{x}(t)\right\rangle_{\mathrm{eq}}$. In experiments, the spectrum is obtained from the record of time evolution of magnetization $M^{x}(t)$. The relation between the autocorrelation and the Fourier transform of time evolution of a quantity $X(t)$ is given by the Wiener-Khinchin relation, that is, the relation between the spectrum of the fluctuation in time of a quantity $X(t)$ and the Fourier transform of the autocorrelation function $\langle X(0) X(t)\rangle$.

We explore the possibility of obtaining the spectrum from the dynamics of the quantity itself, i.e., $\left\langle\Phi_{\beta}|X(t)| \Phi_{\beta}\right\rangle$, from an initial state $\left|\Phi_{\beta}\right\rangle$. In this method, we use the time evolution of the state as in the previous subsection and therefore we can study large systems as well.

\section{A. Wiener-Khinchin theorem}

First, we briefly review the Wiener-Khinchin theorem. For a time-dependent quantity $X(t)$, we define the autocorrelation function $R(t)$ and the spectral density $S(\omega)$ as

$$
R(t) \equiv\langle X(0) X(t)\rangle \equiv \lim _{T \rightarrow \infty} \frac{1}{T} \int_{0}^{T} X(\tau) X(t+\tau) d \tau
$$

and

$$
S(\omega) \equiv \lim _{T \rightarrow \infty} \frac{\left|X^{T}(\omega)\right|^{2}}{T}, \quad X^{T}(\omega) \equiv \int_{0}^{T} X(t) e^{-i \omega t} d t,
$$

respectively. The Wiener-Khinchin theorem tells us that the Fourier transform of the autocorrelation function equals to spectral density:

$$
G(\omega) \equiv \int_{-\infty}^{\infty} R(t) e^{-i \omega t} d t=S(\omega)
$$

Note that we assume that the process $X(t)$ is stationary [31].

\section{B. Dynamics of the magnetization}

Next, we apply the idea of Wiener-Khinchin relation to the Kubo formula. By making use of the relation (33) we obtain
$G(\omega)$ from the spectral density $S(\omega)$. Here it should be noted that $M^{x}(t)$ is an operator and the definition of $\left\langle M^{x}(t)\right\rangle$ is tricky. Definitely, $\left\langle M^{x}(t)\right\rangle_{\mathrm{eq}}$ is time independent, in fact, it is zero in the present case, and therefore we cannot extract $S(\omega)$.

In the following, we propose a method to obtain $G(\omega)$ from $\left\langle M^{x}(t)\right\rangle$ in a quantum-mechanical system. This approach is motivated by the Wiener-Khinchin theorem but we do not use the relation (33) directly. In time-domain methods, we use the notion of the thermal typical state (see above) and we use this notion here once more to derive a formula to obtain $S(\omega)$ from time evolution of $\left\langle M^{x}(t)\right\rangle[32]$.

First we prepare a thermal typical state $\left|\Phi_{\beta}\right\rangle$ as an initial state. The expectation value with this state gives the thermal average at the inverse temperature $\beta$. For $M^{x}(t),\left\langle M^{x}(t)\right\rangle_{\mathrm{eq}}=0$ and the expectation value $\mathrm{E}\left[\left\langle\Phi_{\beta}\left|M^{x}(t)\right| \Phi_{\beta}\right\rangle\right]$ is zero, but, in general, $\left\langle\Phi_{\beta}\left|M^{x}(t)\right| \Phi_{\beta}\right\rangle$ is not zero. When we calculate the time evolution of the sampled state we can extract information for the spectral density

$$
\hat{M}_{\beta}^{T}(\omega) \equiv \int_{0}^{T}\left\langle\Phi_{\beta}\left|M^{x}(t)\right| \Phi_{\beta}\right\rangle e^{-i \omega t} d t .
$$

Although $E\left[\left\langle\Phi_{\beta}\left|\hat{M}^{x}(t)\right| \Phi_{\beta}\right\rangle\right]$ is zero, the expectation value of the spectral density $E\left[\left|\hat{M}_{\beta}^{T}(\omega)\right|^{2}\right]$ is not zero and, as we show below, we can obtain the ESR spectrum from the latter quantity.

Taking the basis to be the eigenstates of the system, $\left\langle\Phi_{\beta}\left|M^{x}(t)\right| \Phi_{\beta}\right\rangle$ is expressed in terms of the random numbers $\left\{\xi_{n}\right\}$ as

$$
\left\langle\Phi_{\beta}\left|M^{x}(t)\right| \Phi_{\beta}\right\rangle=\sum_{m, n} \xi_{m}^{*} \xi_{n} e^{-\beta\left(E_{m}+E_{n}\right) / 2} e^{i\left(E_{m}-E_{n}\right) t}\left\langle m\left|M^{x}\right| n\right\rangle,
$$

where $|n\rangle$ and $E_{n}$ are the eigenvector and its eigenenergy of the system Hamiltonian $\mathscr{H}$ [Eq. (5)]. The Fourier transform $\hat{M}_{\beta}^{T}(\omega)[$ Eq. (34)] is expressed as

$$
\begin{aligned}
\hat{M}_{\beta}^{T}(\omega)= & \sum_{m, n} \xi_{m}^{*} \xi_{n} e^{-\beta\left(E_{m}+E_{n}\right) / 2} e^{-i\left[\omega-\left(E_{m}-E_{n}\right)\right] T / 2} \\
& \times 2 \pi \delta^{T}\left[\omega-\left(E_{m}-E_{n}\right)\right]\left\langle m\left|M^{x}\right| n\right\rangle,
\end{aligned}
$$

where we introduced the function

$$
\delta^{T}(\omega) \equiv \frac{\sin \frac{\omega T}{2}}{\pi \omega} \stackrel{T \rightarrow \infty}{\longrightarrow} \delta(\omega) .
$$

Note that we cannot extend the range of integration as in the AC method [Eq. (14)] because for a given $\left|\Phi_{\beta}\right\rangle$, $\left\langle\Phi_{\beta}\left|M^{x}(t)\right| \Phi_{\beta}\right\rangle^{*}=\left\langle\Phi_{\beta}\left|M^{x}(-t)\right| \Phi_{\beta}\right\rangle$ does not hold in general. For the same number of time steps, this implies that the frequency resolution $\Delta \omega=2 \pi / T$ is twice as large as for the AC method.

As a next step, we calculate the average over the random initial states of the quantity

$$
\begin{aligned}
\left|\hat{M}_{\beta}^{T}(\omega)\right|^{2}= & \sum_{m, n} \sum_{m^{\prime}, n^{\prime}} \xi_{m}^{*} \xi_{n} \xi_{m^{\prime}} \xi_{n^{\prime}}^{*} e^{-\beta\left(E_{m}+E_{n}\right) / 2} e^{-\beta\left(E_{m^{\prime}}+E_{n^{\prime}}\right) / 2} \\
& \times e^{-i\left(\omega-\left(E_{m}-E_{n}\right)\right) T / 2} e^{+i\left(\omega\left(E_{m^{\prime}}-E_{n^{\prime}}\right)\right) T / 2} \\
& \times 4 \pi^{2} \delta^{T}\left[\omega-\left(E_{m}-E_{n}\right)\right] \delta^{T}\left[\omega-\left(E_{m^{\prime}}-E_{n^{\prime}}\right)\right] \\
& \times\left\langle m\left|M^{x}\right| n\right\rangle\left\langle n^{\prime}\left|M^{x}\right| m^{\prime}\right\rangle .
\end{aligned}
$$



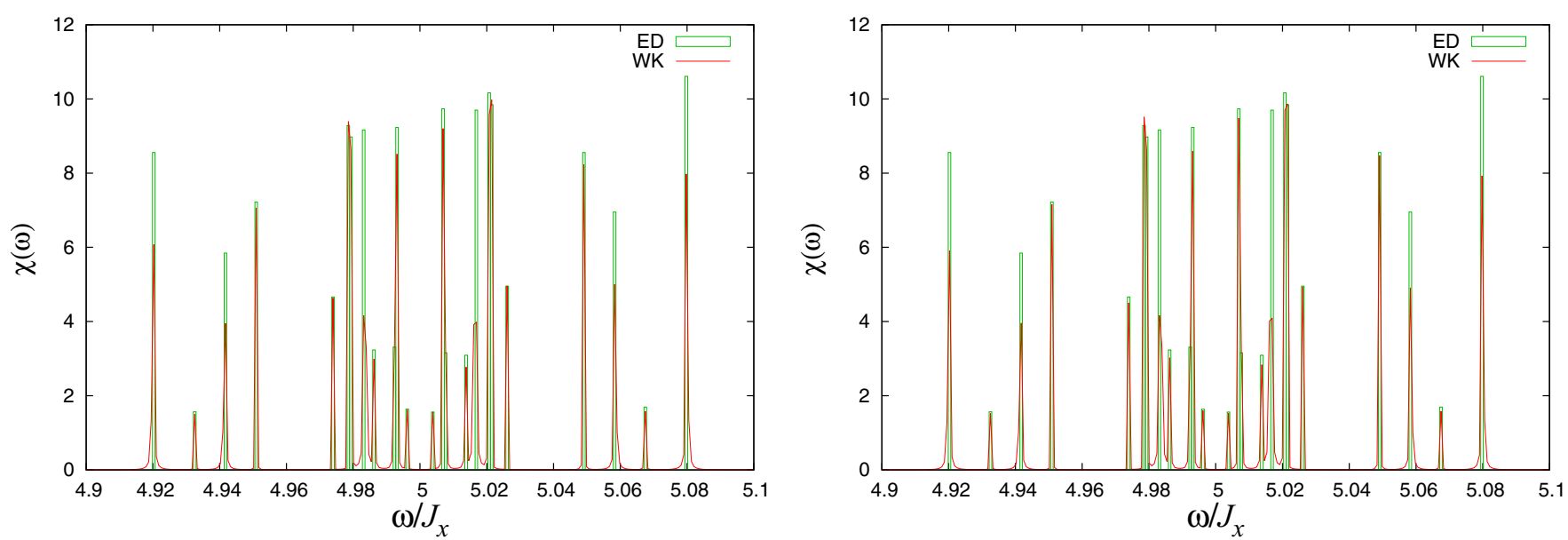

FIG. 3. (Color online) Absorption spectrum obtained by the ED method (green line) and by the WK method (red line): $N=6, \beta^{-1}=100 \mathrm{~K}$, $H=5 \mathrm{~K}$, and the mesh of the frequency $\Delta \omega=2 \pi / 8192$. In the ED method (green line), we take the mesh of the frequency $\Delta \omega=2 \pi / 8192$, which is the interval of the frequency $2 \pi / T$ in the WK method. Left: The Wiener-Khinchin spectrum is obtained by calculating Eq. (39) exactly. Right: The WK spectrum is obtained by averaging 10000 samples of initial thermal typical states.

Using Eq. (20) and the fact that $\left\langle n\left|M^{x}\right| n\right\rangle=0$, we have

$$
\begin{aligned}
E\left[\left|\hat{M}_{\beta}^{T}(\omega)\right|^{2}\right]= & 2 \sigma^{2} T e^{-\beta \omega} \sum_{m, n} e^{-2 \beta E_{n}} 2 \pi \delta^{T} \\
& \times\left[\omega-\left(E_{m}-E_{n}\right)\right]\left|\left\langle m\left|M^{x}\right| n\right\rangle\right|^{2},
\end{aligned}
$$

where we used the relation

$$
\left\{\delta^{T}\left[\omega-\left(E_{m}-E_{n}\right)\right]\right\}^{2} \approx \frac{T}{2 \pi} \delta^{T}\left[\omega-\left(E_{m}-E_{n}\right)\right] .
$$

The spectral density reads

$$
\begin{aligned}
\Sigma_{\beta}(\omega)= & \lim _{T \rightarrow \infty} \frac{E\left[\left|\hat{M}_{\beta}^{T}(\omega)\right|^{2}\right] / Z_{\beta}^{2}}{T} \\
= & 2 \sigma^{2} e^{-\beta \omega} \sum_{m, n} e^{-2 \beta E_{n}} 2 \pi \delta\left[\omega-\left(E_{m}-E_{n}\right)\right] \\
& \times\left|\left\langle m\left|M^{x}\right| n\right\rangle\right|^{2} / Z_{\beta}^{2}
\end{aligned}
$$

where

$$
Z_{\beta}=E\left[\left\langle\Phi_{\beta} \mid \Phi_{\beta}\right\rangle\right]
$$

The Fourier transform of the autocorrelation function is given by

$$
G_{\beta}(\omega)=\sum_{m, n} e^{-\beta E_{n}} 2 \pi \delta\left[\omega-\left(E_{m}-E_{n}\right)\right]\left|\left\langle m\left|M^{x}\right| n\right\rangle\right|^{2} / Z_{\beta} .
$$

By comparing (42) and (44), we obtain a Wiener-Khinchin-like relation for the transverse magnetization:

$$
G_{\beta}(\omega)=2 \sigma^{2} \frac{Z_{\beta / 2}^{2}}{Z_{\beta}} e^{\frac{\beta \omega}{2}} \Sigma_{\beta / 2}(\omega) .
$$

The imaginary part of the dynamical susceptibility of interest is given by

$$
\chi^{\prime \prime}(\omega)=2 \sigma^{2} \frac{Z_{\beta / 2}^{2}}{Z_{\beta}} \sinh \left(\frac{\beta \omega}{2}\right) \Sigma_{\beta / 2}(\omega) .
$$

Here it should be noted that we need to calculate the quantities of $\beta / 2(\operatorname{not} \beta)$ to obtain the ESR spectrum of $\beta$.

In Fig. 3, we show a comparison of the spectrum obtained by the exact diagonalization method and the WK method.

From Fig. 3 it follows that the spectrum is well reproduced without any window procedure, in contrast to Fig. 2 (left) obtained by the AC method. This is attributed to the fact that the finite-time effect (Gibbs oscillation) in the WK method gives positive and smaller artificial peaks in the spectrum (see the Appendix A).

\section{Sample distribution of data}

In principle, methods that are based on the thermal typical state require sampling over the random states to obtain data for equilibrium. However, as mentioned above, the data obtained by the thermal typical state exponentially converge to the thermal equilibrium value as the size of system increases. That is, the deviation decreases proportionally to $D^{-1 / 2}$, where $D$ is the dimension of the Hilbert space. In the AC method, the data are obtained as expectation values of $M^{x} M^{x}(t)$, and for large system the convergence to the equilibrium value is so fast that one initial state suffices to obtain accurate results (see the Supplemental Material in Ref. [28] for the details). On the other hand, the WK method extracts information from the quantity $\hat{M}_{\beta}^{T}(\omega)$ whose expectation value is zero, and therefore we have to investigate the fluctuations of the squared variable $\left|\hat{M}_{\beta}^{T}(\omega)\right|^{2}$ because it is not obvious that these fluctuations will decrease rapidly as $D$ increases.

In this section, we study properties of sample distribution of the data. To characterize the distribution, we study the variance of $\left|\hat{M}_{\beta}^{T}(\omega)\right|^{2}$ :

$$
\operatorname{Var}\left(\left|\hat{M}_{\beta}^{T}(\omega)\right|^{2}\right)=E\left[\left|\hat{M}_{\beta}^{T}(\omega)\right|^{4}\right]-E\left[\left|\hat{M}_{\beta}^{T}(\omega)\right|^{2}\right]^{2}
$$

To this end, we introduce

$$
\tilde{M}_{\beta}^{T}(\omega) \equiv\langle\Phi|A(\omega)| \Phi\rangle
$$


where

$$
A(\omega)=e^{-\beta \mathscr{H} / 2} Y(\omega) e^{-\beta \mathscr{H} / 2}
$$

and

$$
Y(\omega)=\frac{1}{T} \int_{-T / 2}^{T / 2} e^{-i \omega t} e^{i \mathscr{H} t} M^{x} e^{-i \mathscr{H} t} d t .
$$

which is proportional to Eq. (36), i.e., $\widetilde{M}_{\beta}^{T}(\omega)=\hat{M}_{\beta}^{T}(\omega) \times$ $e^{i \omega T / 2} / T$. The reason for shifting the interval from $[0, T]$ to $[-T / 2, T / 2]$ is to assure that $Y(\omega)=Y^{\dagger}(-\omega)$ and

$$
\begin{aligned}
A_{a p} & \equiv A(\omega)_{a p}=\left[A(-\omega)_{p a}\right]^{*}=\left[A^{\dagger}(\omega)_{p a}\right]^{*} \\
& =\frac{\sin \left\{\left[\omega-\left(E_{a}-E_{p}\right)\right] T / 2\right\}}{\left[\omega-\left(E_{a}-E_{p}\right)\right] T / 2}\left\langle a\left|e^{-\beta \mathscr{H} / 2} M^{x} e^{-\beta \mathscr{H} / 2}\right| p\right\rangle,
\end{aligned}
$$

where we choose the states $|a\rangle$ to be the eigenstates of $\mathscr{H}$. With this choice, $\left\langle a\left|M^{x}\right| a\right\rangle=0$ and $A_{a a}=0$, which simplifies the calculations significantly. Using these quantities,

$$
\left|\tilde{M}_{\beta}^{T}(\omega)\right|^{2}=\sum_{a, b, p, q} \xi_{a}^{*} \xi_{b}^{*} \xi_{p} \xi_{q} A_{a p} A_{q b}^{*} .
$$

and, using the identities Eq. (20), the expectation of Eq. (52) is expressed as

$$
E\left[\left|\tilde{M}_{\beta}^{T}(\omega)\right|^{2}\right]=\sum_{a, b} A_{a b} A_{a b}^{*}=4 \sigma^{4} \operatorname{Tr} A A^{\dagger} .
$$

Hereafter, for simplicity of notation but without loss of generality, we choose $2 \sigma^{2}=1$. Note that in Eq. (53), we did not yet include other terms in Eq. (46), i.e., $Z_{\beta}$. But, as we mentioned above, the variances of them are small and thus we only concern the variance Eq. (47).

We calculate the first term in Eq. (47) by making extensive use of the properties of Gaussian random variables. We have

$$
\begin{aligned}
E\left[\left|\tilde{M}_{\beta}^{T}(\omega)\right|^{4}\right]= & \sum_{a, b, c, d} \sum_{p, q, r, s} E\left[\xi_{a}^{*} \xi_{b}^{*} \xi_{c}^{*} \xi_{d}^{*} \xi_{p} \xi_{q} \xi_{r} \xi_{s}\right] A_{a p} A_{q b}^{*} A_{c r} A_{s d}^{*} \\
= & \sum_{a, b, c, d} \sum_{p, q, r, s}\left\{E\left[\xi_{a}^{*} \xi_{p}\right] E\left[\xi_{b}^{*} \xi_{c}^{*} \xi_{d}^{*} \xi_{q} \xi_{r} \xi_{s}\right]+E\left[\xi_{a}^{*} \xi_{q}\right] \mathrm{E}\left[\xi_{b}^{*} \xi_{c}^{*} \xi_{d}^{*} \xi_{p} \xi_{r} \xi_{s}\right]\right. \\
& \left.+E\left[\xi_{a}^{*} \xi_{r}\right] E\left[\xi_{b}^{*} \xi_{c}^{*} \xi_{d}^{*} \xi_{p} \xi_{q} \xi_{s}\right]+E\left[\xi_{a}^{*} \xi_{s}\right] E\left[\xi_{b}^{*} \xi_{c}^{*} \xi_{d}^{*} \xi_{p} \xi_{q} \xi_{r}\right]\right\} A_{a p} A_{q b}^{*} A_{c r} A_{s d}^{*} .
\end{aligned}
$$

The first term in the curly brackets vanishes because $E\left[\xi_{a}^{*} \xi_{p}\right]=\delta_{a, p}$ and $A_{a a}=0$. Interchanging the summation indices $(q, s)$ and $(b, d)$ in the fourth term, respectively, we obtain

$$
\begin{aligned}
E\left[\left|\tilde{M}_{\beta}^{T}(\omega)\right|^{4}\right]= & 4 \operatorname{Tr} A A A^{\dagger} A^{\dagger}+\left|\operatorname{Tr} A^{2}\right|^{2} \\
& +2\left(\operatorname{Tr} A A^{\dagger}\right)^{2}+2 \operatorname{Tr} A A^{\dagger} A A^{\dagger},
\end{aligned}
$$

and using Eq. (53) we finally obtain for the variance

$$
\begin{aligned}
\operatorname{Var}\left(\left|\tilde{M}_{\beta}^{T}(\omega)\right|^{2}\right)= & 4 \operatorname{Tr} A A A^{\dagger} A^{\dagger}+\left|\operatorname{Tr} A^{2}\right|^{2}+\left(\operatorname{Tr} A A^{\dagger}\right)^{2} \\
& +2 \operatorname{Tr} A A^{\dagger} A A^{\dagger} .
\end{aligned}
$$

We want to find bounds to the estimate of the variance Eq. (56). Let us denote the (non-negative) eigenvalues of $A A^{\dagger}$ $\left(A^{\dagger} A\right)$ by $x_{k}^{2}\left(y_{k}^{2}=x_{k}^{2}\right)$, that is, $A A^{\dagger}\left|x_{k}\right\rangle=x_{k}^{2}\left|x_{k}\right\rangle\left(A^{\dagger} A\left|y_{k}\right\rangle=\right.$ $\left.y_{k}^{2}\left|y_{k}\right\rangle\right)$. We assume that the eigenvectors of $A A^{\dagger}$ and $A^{\dagger} A$ are normalized. Then we have

$$
\begin{aligned}
\left(\operatorname{Tr} A A^{\dagger}\right)^{2}= & \left(\sum_{k} x_{k}^{2}\right)^{2} \geqslant \sum_{k} x_{k}^{4}=\operatorname{Tr} A A^{\dagger} A A^{\dagger}, \\
\operatorname{Tr} A A^{\dagger} A^{\dagger} A & =\sum_{k, l} x_{k}^{2} y_{l}^{2}\left|\left\langle x_{k} \mid y_{l}\right\rangle\right|^{2} \leqslant \sum_{k, l} x_{k}^{2} y_{l}^{2} \\
& =\left(\operatorname{Tr} A A^{\dagger}\right)\left(\operatorname{Tr} A^{\dagger} A\right)=\left(\operatorname{Tr} A A^{\dagger}\right)^{2} .
\end{aligned}
$$

As $(X, Y) \equiv \operatorname{Tr} X^{\dagger} Y$ defines a scalar product, by the Schwarz inequality $\left|\left(A^{\dagger}, A\right)\right|^{2}=\left|\operatorname{Tr} A^{2}\right|^{2} \leqslant\left|\operatorname{Tr} A A^{\dagger}\right|^{2}$. Noting that the last term in Eq. (56) cannot be negative, putting all this together we find

$$
1 \leqslant 1+\frac{4 \operatorname{Tr} A A A^{\dagger} A^{\dagger}+\left|\operatorname{Tr} A^{2}\right|^{2}+2 \operatorname{Tr} A A^{\dagger} A A^{\dagger}}{\left(\operatorname{Tr} A A^{\dagger}\right)^{2}} \leqslant 8 .
$$

Thus, we find the variance is bounded as

$$
1 \leqslant \frac{\operatorname{Var}\left(\left|\hat{M}_{\beta}^{T}(\omega)\right|^{2}\right)}{E\left[\left|\hat{M}_{\beta}^{T}(\omega)\right|^{2}\right]^{2}} \leqslant 8,
$$

indicating that in the WK method, it is necessary to average over different initial states, also for large systems. This is in sharp contrast to the case of the AC method in which the variance vanishes exponentially with the system size. But Eq. (60) also indicates that the variance is bounded, independent of the system size, and therefore we can obtain good estimates for the mean from finite samples.

We emphasize that the variance of the WK method does not depend on $D$, that is, the distribution shows a kind of typicality. Thus we can obtain the correct mean with aimed precision by sampling, regardless of the system size.

\section{DISTRIBUTION OF AVERAGE VALUE AND CONVERGENCE IN DISTRIBUTION}

In this section we investigate the sample distribution of $\chi(\omega)$ data. For concreteness, we choose the value of $\omega$ to be $4.9916 \mathrm{~K}$ at which the spectrum has a maximum (peak). 

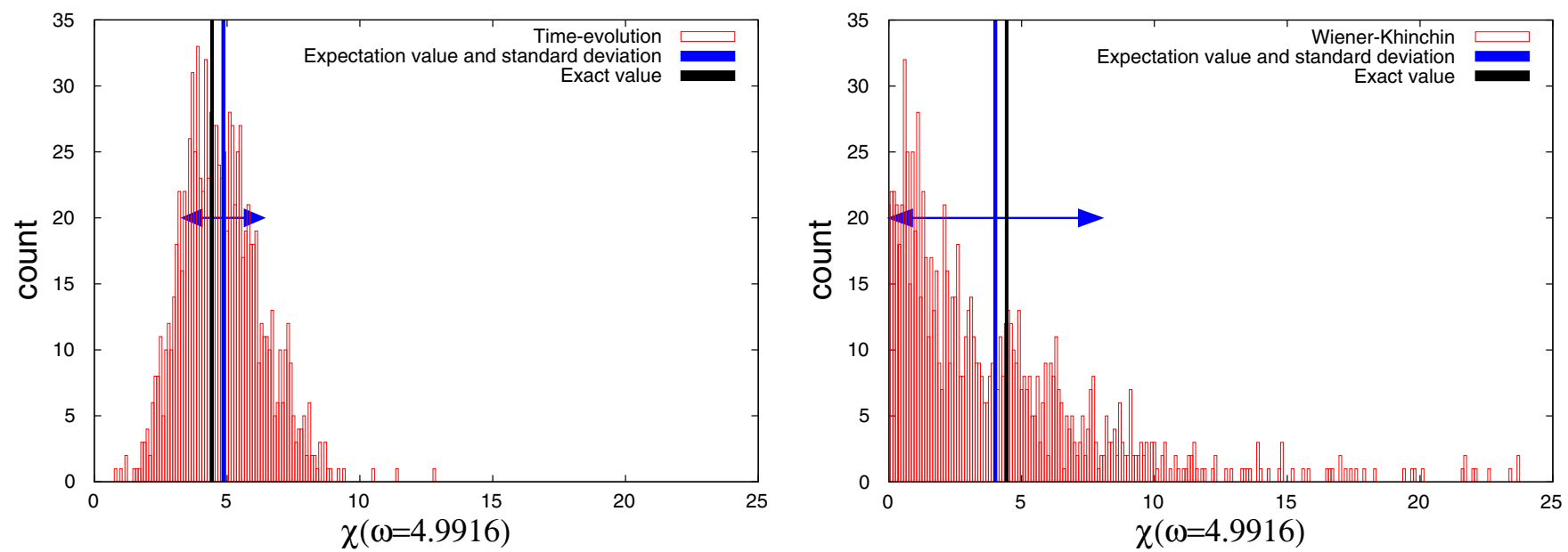

FIG. 4. (Color online) Left: The distribution of values over 1000 samples obtained by the AC method at $\omega=4.9916 \mathrm{~K}$ for $N=10$, $\beta^{-1}=100 \mathrm{~K}$. The mean $(=4.8508 \mathrm{~K})$ is given by the blue line, and the standard deviation $(=1.5049 \mathrm{~K})$ is shown by the arrow. The exact value is denoted by the black solid line whose $\omega=4.4666 \mathrm{~K}$. Right: The distribution of values over 1000 samples obtained by the WK method at $\omega=4.9916 \mathrm{~K}$ for $N=10, \beta^{-1}=100 \mathrm{~K}$. The mean $(=4.01579 \mathrm{~K})$ is given by the blue line, and the standard deviation $(=3.9749 \mathrm{~K})$ is shown by the arrow. The exact value is denoted by the black solid line whose $\omega=4.4666 \mathrm{~K}$.

First we show the distribution of data in the $\mathrm{AC}$ method for the system $N=10$, where $D=1024$ and $D^{-1}$ is rather small. The histogram of the data is given in Fig. 4 (left). The exact value is denoted by the black line. The mean of the distribution is shown by the bold blue line. The standard deviation (square root of the variance) is given by the arrow. Clearly, Fig. 4 (left) shows that the data are distributed around the correct value with $\mathrm{RSD}=0.31025 \mathrm{~K}$. As we already mentioned several times, as the size of the system increases the variance will vanish as $D^{-1}$.

The histogram obtained by the WK method is depicted in Fig. 4 (right). In contrast to the AC method, the distribution is of the exponential type. From the figure, we find the variance is about the same as the average, i.e., $\mathrm{RSD}=0.98982 \mathrm{~K}$. This value corresponds to the lower bound of the estimation (60). This fact will be discussed in more detail below.

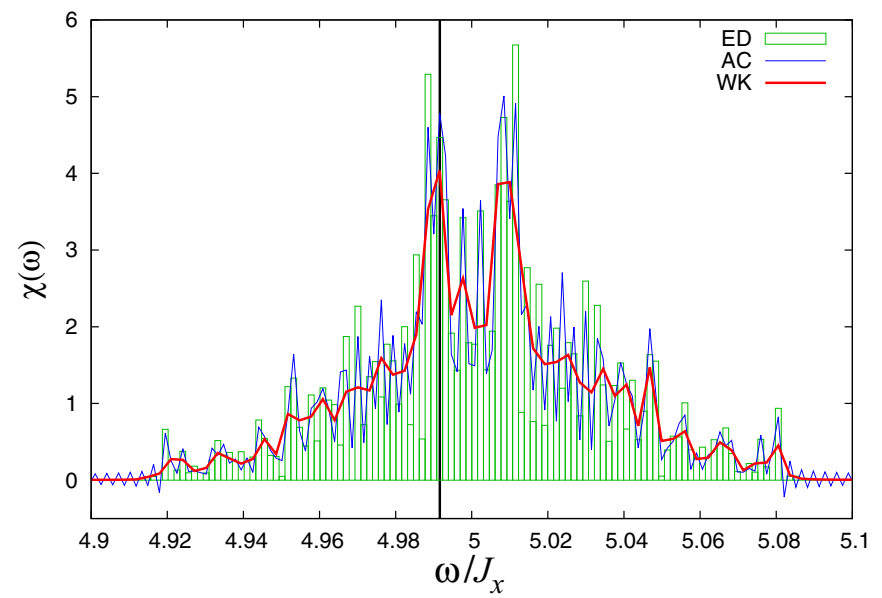

In Fig. 5, we present the spectra for $N=10$ obtained by the both methods compared with the exact one obtained by the ED method. Because we have the exact eigenstates and eigenvalues, we computed the results of $\mathrm{AC}$ and WK methods by employing Eqs. (36) and (44) using the exact eigenstates and eigenvalues (not by sampling).

Both methods seem to reproduce the exact one approximately. But the spectra by the AC method are considerably suffering from the effect of finite observation time and show large fluctuations in both cases where $n_{t}$ is small (left) and large (right). Especially the existence of the negative peaks seen in Fig. 5 (right) is a significant disadvantage to the AC method as mentioned before. On the other hand, the WK method automatically smears out the oscillations and gives rather good agreement. Of course, the negative peaks never appear by definition.

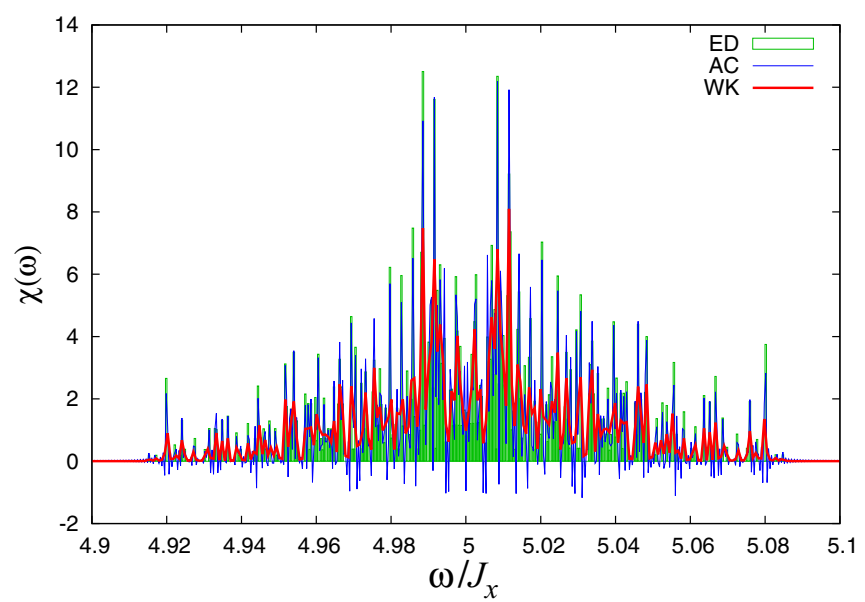

FIG. 5. (Color online) Left: Spectra obtained by the AC method (blue line) by the WK method (red line) with $n_{t}=4096, \beta^{-1}=100 \mathrm{~K}$. The column histogram is obtained by the ED method. The mesh of the frequency $2 \pi / 4096$ for the WK method and $\pi / 4096$ for the AC method and the ED method. The line $\omega=4.9916 \mathrm{~K}$ is denoted by the black line. Right: $\beta^{-1}=100 \mathrm{~K}, n_{t}=16384$, and the mesh of the frequency $2 \pi / 16384$ for the WK method and $\pi / 16384$ for the AC method and the ED method. 
In the case of the AC method, the obtained values have a narrow distribution around the mean, and it is known that the variance decreases as $D^{-1}$. In this sense, the AC method has an advantage. But the WK method also gives correct estimates by sampling and the obtained shape of the spectrum is close to the exact values.

\section{A. Special case 1: Energy gap selection}

In the previous section, we found that the relative variance is close to 1 , the minimum value of the bound Eq. (60). In this subsection, we consider the reason of this fact. To derive Eq. (60), we considered the general case. But we can also use the fact that $A_{a p}$ [Eq. (51)] is almost zero except when $\omega \simeq E_{a}-E_{p}$. If we assume that there is a unique set $(n, l, m)$ which satisfies the conditions $E_{m}-E_{n}=E_{m}-E_{l}=\omega$ and $\left\langle m\left|M^{x}\right| n\right\rangle\left\langle m\left|M^{x}\right| l\right\rangle \neq 0$, then all terms are negligibly small except for the term $\left(\mathbf{T r} A A^{\dagger}\right)^{2}$. Therefore

$$
\frac{\sqrt{\operatorname{Var}\left(\left|\hat{M}_{\beta}^{T}(\omega)\right|^{2}\right)}}{E\left[\left|\hat{M}_{\beta}^{T}(\omega)\right|^{2}\right]} \simeq \frac{\left(\operatorname{Tr} A A^{\dagger}\right)^{2}}{\left(\operatorname{Tr} A A^{\dagger}\right)^{2}}=1,
$$

which is indeed what we have observed in our actual calculations.

\section{B. Special case 2: Isolated $\delta$ function}

We give the variance of the WK method for the case where the spectrum consists of well-separated $\delta$ functions which are given only by a single resonant pair of states, that is, the nonresonance condition that if $E_{m}-E_{n}=E_{m^{\prime}}-E_{n^{\prime}}$, then ( $m=m^{\prime}$ and $\left.n=n^{\prime}\right)$ or $\left(m=n\right.$ and $\left.m^{\prime}=n^{\prime}\right)$ is satisfied. Then, we can use the fact that approximately $\delta^{T}(x)=\delta(x)$ and we have

$$
\begin{aligned}
E\left[\left|\hat{M}_{\beta}^{T}(\omega)\right|^{2}\right]= & 2 \sigma^{2} T \mathrm{e}^{-\beta \omega} e^{-2 \beta E_{n}} \\
& \times 2 \pi \delta^{T}\left[\omega-\left(E_{m}-E_{n}\right)\right]\left|\left\langle m\left|M^{x}\right| n\right\rangle\right|^{2} .
\end{aligned}
$$

The variance is estimated by making use of the relation:

$$
E\left[\xi_{m}^{*} \xi_{n} \xi_{m^{\prime}} \xi_{n^{\prime}}^{*} \xi_{k}^{*} \xi_{l} \xi_{k^{\prime}} \xi_{l^{\prime}}^{*}\right]=E\left[\left(\xi_{m}^{*} \xi_{m}\right)^{2}\left(\xi_{n}^{*} \xi_{n}\right)^{2}\right] \simeq 4
$$

as

$$
E\left[\left|\hat{M}_{\beta}^{T}(\omega)\right|^{4}-E\left[\left|\hat{M}_{\beta}^{T}(\omega)\right|^{2}\right]^{2}\right] \simeq 3 E\left[\left|\hat{M}_{\beta}^{T}(\omega)\right|^{2}\right]^{2} .
$$

Thus, the relative variance is 3 , a result which does not depend on $D$.

In the AC method, the variance of sampled amplitude of the isolated $\delta$ functions is the same as the expectation value for the present case and RSD $\geqslant 1$. However, the isolated $\delta$ function in the spectrum is only relevant in small systems. For large systems with large $D$, the amplitude of the isolated $\delta$ function is small and we can ignore it.

\section{THE TOTAL AMPLITUDE OF THE SPECTRUM}

The total amplitude of the spectrum, or the intensity, i.e., the integral over the absorption spectrum [Eq. (2)], is given by

$$
I^{x}=\int_{0}^{\infty} I^{x}(\omega) d \omega .
$$

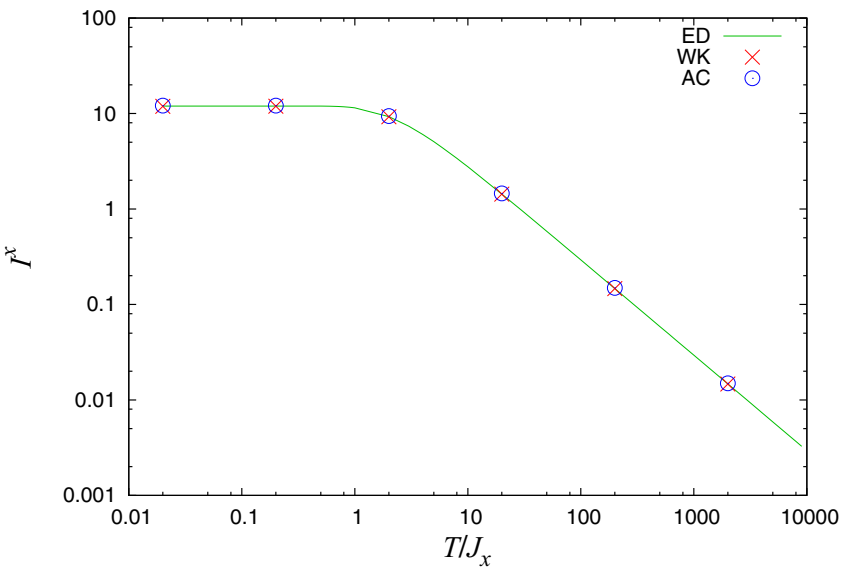

FIG. 6. (Color online) Comparison of the intensities of the absorption as a function of the temperature obtained by the WK method (red cross) and by the AC method (blue open circle). The green solid curve is the exact value: $N=6, H=5 \mathrm{~K}$.

It is one of the fundamental pieces of information obtained in ESR experiments. Its temperature dependence has been calculated for the single molecular magnet $\mathrm{V}_{15}$ [18]. In this subsection, we demonstrate that $I^{x}$ obtained by the WK method correctly reproduces the exact ED results. Details of the analysis are given in the Supplemental Material in Ref. [29]. In the WK method, a $\delta$ function is represented by the sinc function,

$$
\delta(\omega) \rightarrow \frac{1}{T}\left(\frac{\sin \omega T / 2}{\pi \omega}\right)^{2} .
$$

Because of the relation

$$
\frac{1}{T} \int_{-\infty}^{\infty}\left(\frac{\sin \omega T / 2}{\pi \omega}\right)^{2} d \omega=\frac{1}{2 \pi},
$$

the intensity $I^{x}$ is obtained correctly by analytical integration. However, the DFT yields the spectrum at discrete points $\omega=2 k \pi / T, \quad k=0,1,2, \cdots, n_{t}-1$ only. Thus, the integral over the $\omega$ is given by a discrete sum. In the Supplemental Material in Ref. [29], we demonstrate that the discrete sum agrees with the analytical result. We also show that for the AC method in which the discrete points are given by $\omega=$ $k \pi / T, \quad k=-n_{t}, \ldots,-1,0,1,2, \ldots, n_{t}-1$ we recover the analytical result of the intensity, in spite of Gibbs oscillations. We calculate the results of the AC and WK methods by evaluating the formulas for the expectation value by using the exact eigenvalues and eigenstate, as we did for Figs. 2 and 3 (left), i.e., without sampling over thermal typical states. In Fig. 6, we show that both the AC and WK methods reproduce the results of the ED method.

\section{APPLICATIONS TO LARGE SYSTEMS}

As an example of application of the methods to larger systems, we compute the spectrum by the AC and WK method for $N=16$ with $n_{t}=16384$. For this size, we can still compute all eigenvalues and eigenstates, hence we know the positions of the eigenfrequencies and the corresponding amplitudes. The ensemble of $\delta$ functions is turned into a 

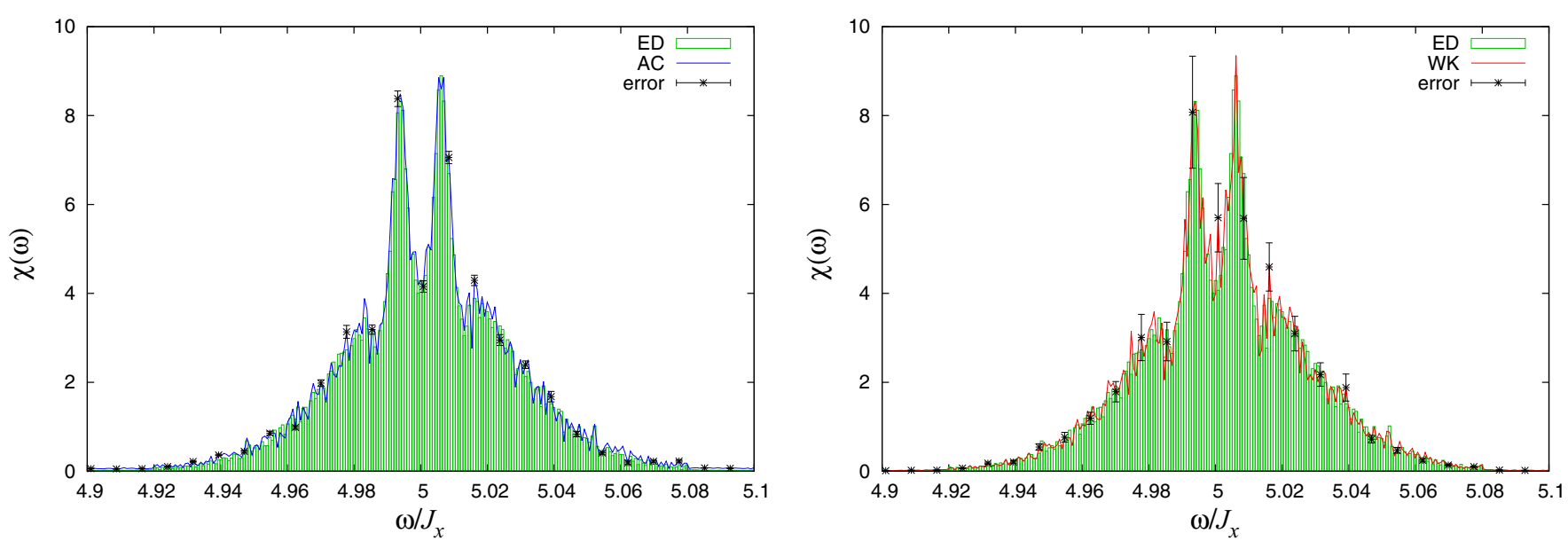

FIG. 7. (Color online) Left: Spectrum for $N=16, \beta^{-1}=100 \mathrm{~K}$. The histogram is obtained by exact diagonalization. The points with error bars are the data obtained by the AC method with 10 samples and the time interval $[0, T=16384 \times d t], d t=0.5$. Right: Results of the WK method with 52 samples over the same time interval.

histogram by using a frequency mesh $\Delta \omega=2 \pi / T,[T=$ $16384 \times d t(=0.5)]$.

In Fig. 7 (left), we present a comparison between the spectra obtained by ED and AC methods. The data obtained by the AC method shows sharp features because we did not use a window in the DFT procedure. As the statistical fluctuations on the autocorrelation are small, 10 samples suffice to find almost perfect agreement with the ED result. Therefore, we may conclude that as the size of the system increases, the effects of finiteness of the time interval reflected in, e.g., Gibbs oscillations seems to be small, whereas we found serious effects in the case $N=6,10$.

In Fig. 7 (right), we show the comparison between the spectra obtained by the ED and WK method. In the WK method, we took 52 samples, and we did not use a window function for the DFT procedure. Here we again find a good agreement but the variance of ensemble average is about the same as the mean as we found in the case $N=10$. We conclude that for $N=16$, the results of both methods are in good agreement with those of ED.

With above observation, we also obtained spectra for $N \geqslant 20$ by the AC method. Up to now, we adopted the periodic boundary conditions (PBC). For a change and also because in actual materials open boundary conditions (OBC) are more natural, we show results for $N=20$ obtained for both boundary conditions. In Fig. 8 (left), the red curve (circles) is the spectrum obtained with PBC and the blue curve with triangles is that with $\mathrm{OBC}$. We find that the spectrum of $\mathrm{OBC}$ has a shaper double peak in the center, but the global shape is similar in both cases. Finally, we present spectra for $N=24,26$ for OBC in Figs. 8 (center) and 8 (right), respectively. We used $n_{t}=16384$ with $d t=0.5$ for $N=20$ and $n_{t}=8192$ with $d t=0.5$ for $N=24,26$ and did not use any windowing procedure.

The size dependence of the separation between double peaks $\Delta \omega$ in the large size limit is an interesting issue and has been discussed in the previous paper [14]. Following the previous paper, we study the separation of the Fourier transform of the autocorrelation function

$$
S^{x x}(\omega, H)=\int_{-T}^{T}\left\langle M^{x} M^{x}(t)\right\rangle_{\mathrm{eq}} e^{-i \omega t} d t .
$$

For this quantity, in the present system, the relation $S^{x x}(\omega, H)=S^{x x}(\omega-\gamma H, 0)$ holds, where $\gamma=g \mu_{B} / \hbar$. And thus, we study this quantity at $H=0$, which is faster in calculation than the case of nonzero $H$. At high temperatures and when $H$ is sufficiently larger than half of the separation $H_{c} \equiv \Delta \omega / 2 \gamma, \chi^{\prime \prime}(\omega, H) \sim \omega \beta S^{x x}(\omega, H) / 2$, and the separation in $S_{x x}(\omega, H)$ at large $H$ is almost the same as that in $\chi^{\prime \prime}(\omega, H)$. In $\chi^{\prime \prime}(\omega, H)$ for $H<H_{c}$, the double-peak structure
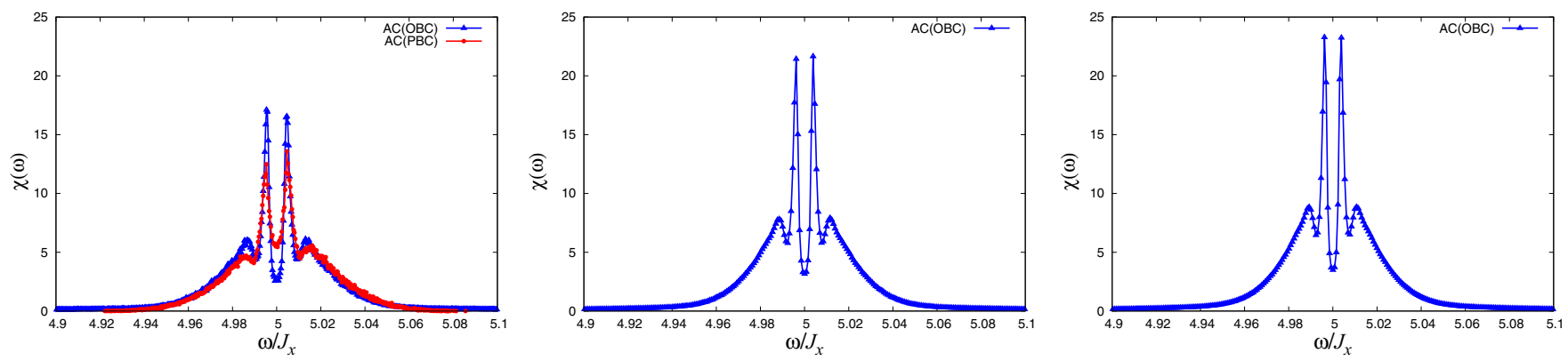

FIG. 8. (Color online) Spectrum obtained by the AC method with the open boundary condition (blue line) at infinite temperature $\beta^{-1}=$ 100 K. Left: $N=20$. The red line denotes the spectrum with the periodic boundary condition. Center: $N=24$. Right: $N=26$. 


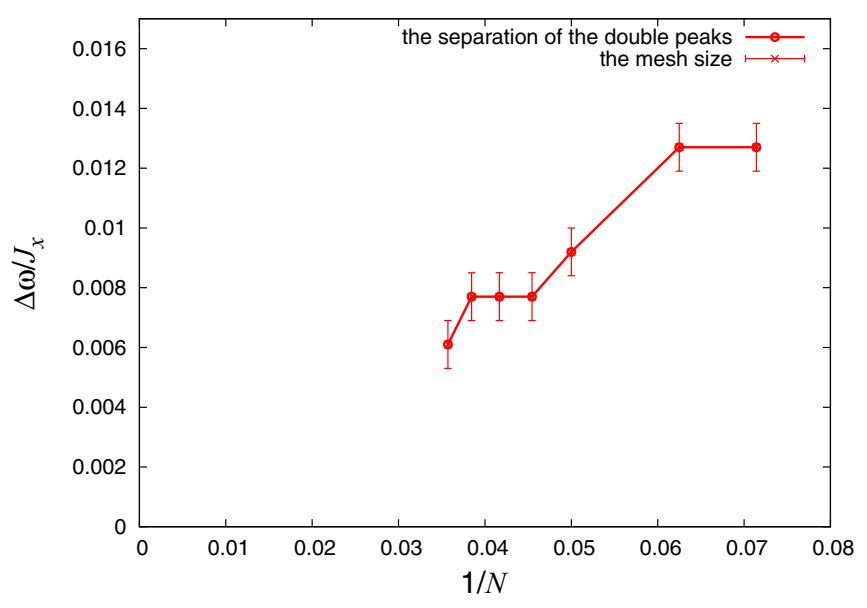

FIG. 9. (Color online) Size dependence of the separation of the double peaks. The red points correspond to the system size $N=$ $14,16,20,22,24,26$, and 28 from right to left. The error bars denote the mesh size of $\Delta \omega$ determined by the observation time $T$.

is deformed because of the factor $1-e^{-\beta \omega}$ in Eq. (1) and the structure of the so-called antiferromagnetic resonance appears. Size dependence of the separation of the double peaks is depicted in Fig. 9. The error bar in the figure denotes the mesh size of $\Delta \omega$ determined by the observation time. Here we find that the separation roughly decreases with the size as $1 / N$ at least up to $N=22$, and that the separations of $N=22,24$, and 26 are almost the same. But at $N=28$ it again decreases. Thus, at this moment, we cannot conclude how the separation behaves in the large size limit. This problem will be studied elsewhere.

\section{SUMMARY AND DISCUSSION}

We have studied time-domain methods to compute the ESR spectrum through sampling of thermal typical states. Although it has been known that the autocorrelation function can be obtained with a few samples thanks to the typicality property, practical aspects of these calculations have not yet been explored.

We proposed a method to obtain the spectrum from the motion of $M^{x}$ by making use of the Wiener-Khinchin-like relation. The dynamics of magnetization from sampled initial states provides an estimate the spectrum density. But in the quantum equilibrium state the expectations value of $M^{x}(t)$ and the sampled data for the spectrum density $M_{\beta}^{x}(\omega)$ are zero. Then, we take thermal typical states as initial states and obtain the average of squared spectrum density $\left|M_{\beta}^{x}(\omega)\right|^{2}$, i.e., the Fourier transform of the autocorrelation function. To this end, we proposed a quantum mechanical version of the Wiener-Khinchin relation. However, it should be mentioned that the WK method does not directly relate to the experimental situation. Because $\left\langle M_{\beta}^{x}(\omega)\right\rangle_{\mathrm{eq}}=0$, the averaged squared spectrum density $\left|M_{\beta}^{x}(\omega)\right|^{2}$ does not converge to a mean value but has a finite distribution, also for large systems. Therefore the convergence property of the thermal typical state does not apply in this case. We studied statistical properties of the distribution and found that form of the distribution converges in the sense of typicality. We derived bounds on the relative variance of the distribution.

In time domain methods the effect of finite observation time is an important issue. We studied this aspect in detail for both the AC and WK method. It was found that for small systems, the AC method is suffering from severe effect of finiteness of $T$ while in the WK method the effect is practically suppressed. The WK and AC methods give complementary information for the effects of finite $T$ and a comparison between them is useful to confirm that the obtained result is not affected by the finiteness observation time. We also found that for both the AC and WK methods, the effect of finiteness of $T$ decreases with increasing system size. As the system size increases, the efficiency of the AC method increases, too.

We presented the spectrum for a one-dimensional XXZ chain up with to $N=26$ spins, where we found the doublepeak structure clearly. In order to study size dependence of the separation of the double peak, we calculated the Fourier transform of the autocorrelation function, $S_{x x}(\omega, H=0)$, which is almost the same as the $\chi(\omega+\gamma H, H)$ at high temperatures when $H$ is larger than the separation. We obtained the data up to $N=28$. It was discussed in a previous paper [14] that the shape of spectrum reflects of the dynamical property of the force for spin dynamics, i.e., torque, and whether the double-peak structure remains in large sizes or not gives an important information for the autocorrelation of the torque. The present study for longer chains gives data for the size dependence. But from the data, we could not conclude yet whether it disappears in the thermodynamic limit or not. The study on longer chain is necessary, but at the present computer facilities it would take very long time, and we will study this problem elsewhere separately with a faster computer.

In our numerical work, we focused on chains with an even number of spins. The ESR spectra of chains with an odd number of spins show behavior that qualitatively differs from the one of chains with an even number of spins, meriting a study in its own right. As the focus of the present paper was on time-domain methods rather than on specific applications, we relegate this study to future research.

In summary, in this paper, we proposed the new method to compute the ESR spectrum by making use of mathematical relations discussed in Sec. III. We have studied one particular time-domain method based on the Wiener-Khinchin theorem but there are many other ways to obtain the spectrum density by making use of relations similar to Wiener-Khinchin theorem. For example, if we calculate $E\left[\left\langle\Phi_{\beta}\left|M^{x}\right| \Phi_{\beta}\right\rangle\left\langle\Phi_{\beta}\left|M^{x}(t)\right| \Phi_{\beta}\right\rangle\right]$, we can obtain a similar expression. Studying the relation among them is an interesting problem for future research.

\section{ACKNOWLEDGMENTS}

The present work was supported by Grants-in-Aid for Scientific Research C (25400391) from Ministry of Education, Culture, Sports, Science and Technology (MEXT) of Japan and the Elements Strategy Initiative Center for Magnetic Materials under the outsourcing project of MEXT. The numerical calculations were supported by the supercomputer center of Institute of Solid State Physics (ISSP) of Tokyo University. We also acknowledge the JSPS Core-to-Core Program: Nonequilibrium dynamics of soft matter and information. 

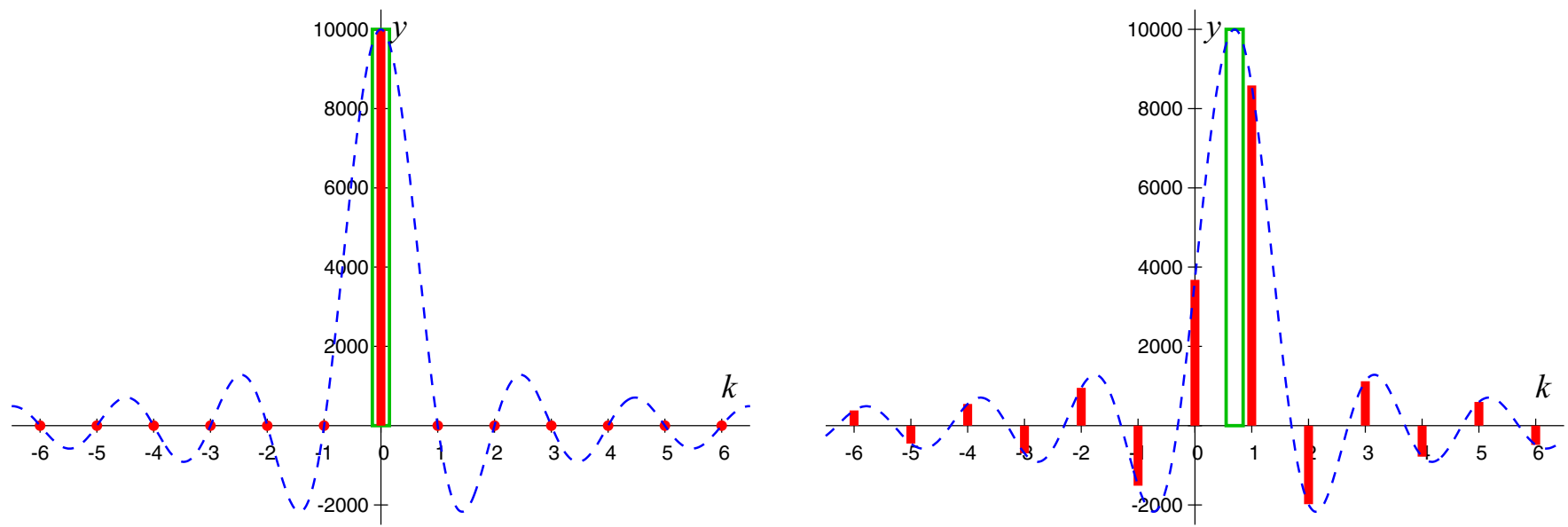

FIG. 10. (Color online) These figures illustrate the forms of the DFT spectra obtained by the AC method. The dashed blue curves denote the spectra of $|\hat{H}(\omega)|: n=10000, \Delta=0.5$. Note that these curves are just theoretical auxiliary lines and not the DFT spectra we can observe in practice. Left: The true $\delta$ peak denoted by the green box is located at $k=0$, which is one of the mesh points. In this case, the DFT spectrum (red rods) appears as a single peak and reproduces the original $\delta$ peak precisely. Right: The true $\delta$ peak is located at $k=0.7$, which is between the mesh points. In this case, the DFT spectrum, which is observed only on the mesh points, shows oscillation including negative peaks.

APPENDIX: EFFECT OF FINITENESS OF THE OBSERVATION TIME DOMAIN AND WINDOW FUNCTION

Because the observation time is finite $[-T, T]$ and we have a finite number of points in time, the discrete Fourier transform (DFT) gives amplitude at the discrete points

$$
\omega_{k}=\Delta \omega \times k, \quad \Delta \omega=\frac{\pi}{T}, \quad k: \text { integer. }
$$

On the other hand, the resonance points are not necessarily at those points. If the spectrum has a $\delta$ function at $\omega$, this is expressed by several point at the discrete points in the spectrum obtained by the DFT [30]. We study this phenomenon for the $\mathrm{AC}$ and WK methods.

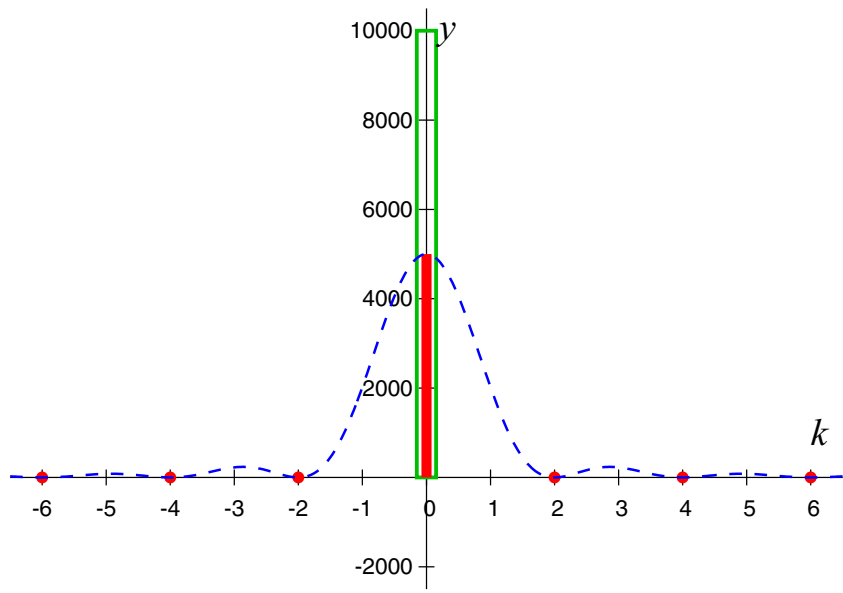

For a time sequence of data $\left\{f_{k}\right\}_{k=-\infty}^{\infty}$ such as the autocorrelation function, the spectrum of the process is given by the DFT:

$$
\begin{gathered}
F(\omega)=\Delta \sum_{k=-\infty}^{\infty} f_{k} e^{-i \omega k \Delta}\left[\simeq \int_{-\infty}^{\infty} f(t) e^{-i \omega t} d t\right], \\
f_{k}=\frac{1}{2 \pi} \int_{-\pi / \Delta}^{\pi / \Delta} F(\omega) e^{i \omega k \Delta} d \omega,
\end{gathered}
$$

where $\Delta$ is the sampling interval. For a set with a finite number, say, $2 n$, the transformation reads

$$
\widetilde{F}(\omega) \equiv \Delta \sum_{k=-n}^{n-1} f_{k} e^{-i \omega k \Delta}=\Delta \sum_{k=-\infty}^{\infty} f_{k} h_{k} e^{-i \omega k \Delta},
$$

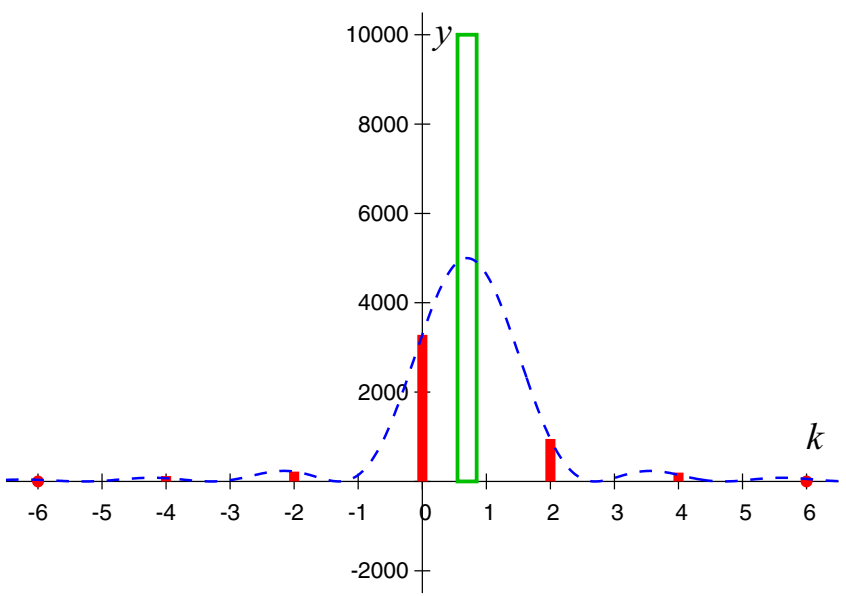

FIG. 11. (Color online) These figures illustrate the forms of the DFT spectra obtained by the WK method. The dashed blue curves denote the spectra of $\frac{1}{4 T}|\hat{H}(\omega)|^{2}: n=10000, \Delta=0.5$. Left: The true $\delta$ peak denoted by the green box is located at $k=0$, which is one of the mesh points. In this case, the DFT spectrum (red rods) appears as a single peak and its height is half of that of the original $\delta$ peak. But it is noted that the interval of the mesh points in the WK method is twice as wide as that in the AC method, and so we can consider the area of the peak is conserved. (See the next subsection in detail.) Right: The true $\delta$ peak is located at $k=0.7$, which is between the mesh points. In this case, the DFT spectrum, which is observed only on the mesh points, shows oscillation. But in contrast to the AC method, the spectrum never include the negative peaks. 

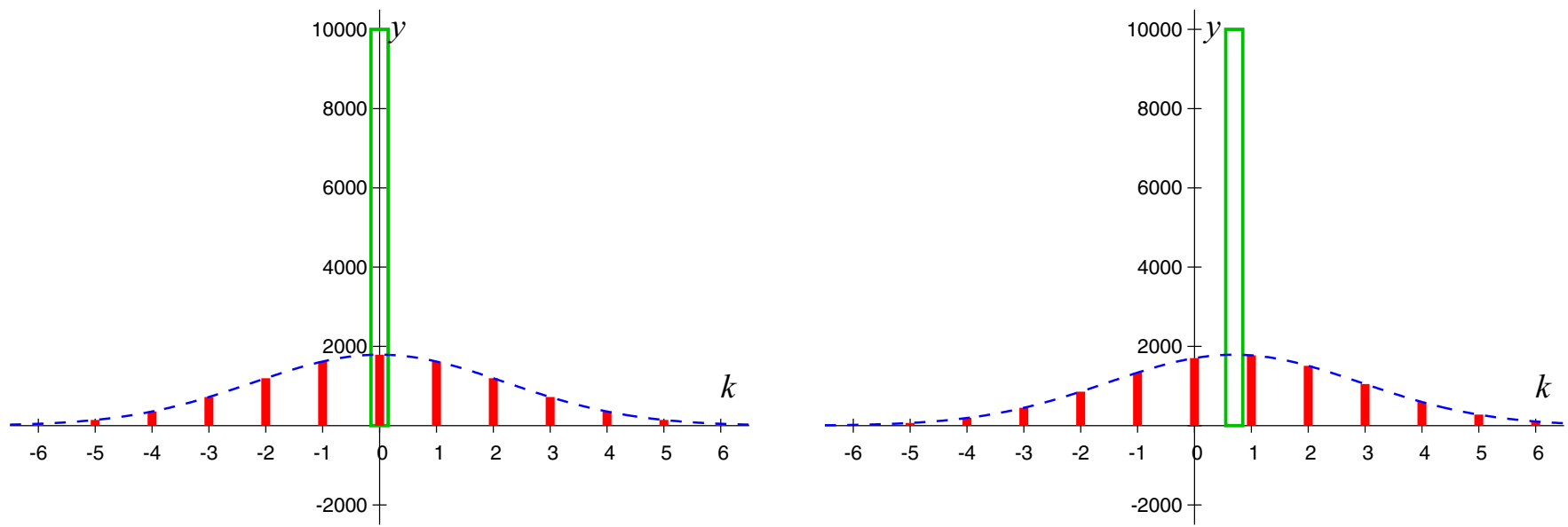

FIG. 12. (Color online) These figures illustrate the forms of the DFT spectra obtained by the AC method with a Gaussian window. The dashed blue curves denote the spectra $|G(\omega)|: n=10000, \Delta=0.5$, and $\alpha=7$. Left: The true $\delta$ peak denoted by the green box is located at $k=0$, which is one of the mesh points. In this case, the DFT spectrum (red rods) appears as a Gaussian-like function with mean 0. Its variance is determined by the artificial $\alpha$. Right: The true $\delta$ peak is located at $k=0.7$, which is between the mesh points. Even in this case, the oscillation is not observed due to the masking effect of the Gaussian window.

where

$$
h_{k}= \begin{cases}1 & k=0, \pm 1, \pm 2 \ldots, \pm(n-1),-n \\ 0 & \text { otherwise. }\end{cases}
$$

Expression Eq. (A4) can be written as a convolution:

$$
\begin{aligned}
\widetilde{F}(\omega) & =\frac{1}{2 \pi} \int_{-\pi / \Delta}^{\pi / \Delta} F\left(\omega^{\prime}\right) H\left(\omega-\omega^{\prime}\right) d \omega \\
& =\frac{1}{2 \pi} \int_{-\pi / \Delta}^{\pi / \Delta} F\left(\omega-\omega^{\prime}\right) H\left(\omega^{\prime}\right) d \omega,
\end{aligned}
$$

where $H(\omega)$ is the DFT of $\left\{h_{k}\right\}_{k}$

$$
H(\omega)=\Delta \sum_{k=-n}^{n-1} e^{-i \omega k \Delta}=\Delta e^{i \frac{\omega \Delta}{2}} \frac{\sin (\omega n \Delta)}{\sin (\omega \Delta / 2)},
$$

indicating that the spectrum we want is deformed by

$$
\hat{H}(\omega) \equiv \Delta \frac{\sin (\omega n \Delta)}{\sin (\omega \Delta / 2)} .
$$

In Fig. 10 (left), the blue dashed curve shows the modulus of Eq. (A8). If $F(\omega)$ is a $\delta$ function $\delta\left(\omega-\omega_{\text {peak }}\right)$ the spectrum obtained by DFT is

$$
\widetilde{F}(\omega)=\frac{1}{2 \pi} H\left(\omega-\omega_{\text {peak }}\right)
$$

for $\omega$ given by the mesh points $\left\{\omega_{k}\right\}$. If $\omega_{\text {peak }}$ is one of the mesh points, then the DFT spectrum has a single peak. However, if $\omega_{\text {peak }}$ is located in an interval of mesh points, then the DFT spectrum has several peaks as shown in Fig. 10 (right). The oscillation of $H(\omega)$ even gives peaks with negative amplitude.
The red rods show how the $\delta$ function in the correct spectrum at the position of green box appears in finite discrete spectrum in the AC and WK methods. In contrast to the AC method, in the WK method the spectrum is squared and the spectrum is given by $|\widetilde{F}(\omega)|^{2}$, hence the spectrum is positive by construction. Although Gibbs oscillations are present, they give a width due to the finite time window and do not affect the spectrum much. In Figs. 10 and 11, we show comparison of $\hat{H}(\omega)$ and $|\hat{H}(\omega)|^{2}$, respectively. Note that in the WK method, we can obtain the discrete spectrum only at the points of even $k$ 's.

In order to avoid these apparent negative peaks, a window function, often a Gaussian, is introduced:

$$
\int_{-T}^{T} f(t) e^{-i \omega t} e^{-\frac{1}{2}\left(\alpha \frac{t}{T}\right)^{2}} d t=2 \operatorname{Re}\left[\int_{0}^{T} f(t) e^{-i \omega t} e^{-\frac{1}{2}\left(\alpha \frac{t}{T}\right)^{2}} d t\right] .
$$

This treatment replaces $f_{k}$ by

$$
g_{k}=h_{k} e^{-\frac{1}{2}\left(\alpha \frac{k}{n}\right)^{2}}, \quad k=0, \pm 1, \pm 2, \ldots
$$

The resulting spectrum $|G(\omega)|$ is depicted in Fig. 12 .

The parameter $\alpha$ determines the artificial resolution of the spectrum. Within this resolution, the Gibbs oscillations are smeared out, hence we can reproduce the spectrum as discussed in Sec. II C.

The width of the Gaussian window $\alpha$ is known to be taken as

$$
e^{-\frac{1}{2}\left(\alpha \frac{n}{n}\right)^{2}}=\varepsilon \rightarrow \alpha^{2}=-2 \ln \varepsilon,
$$

where $\varepsilon$ is a number of the order of the smallest number of the computer resolution, say, $\varepsilon=10^{-12}$.
[1] S. Eggert and I. Affleck, Phys. Rev. B 46, 10866 (1992); Phys. Rev. Lett. 75, 934 (1995); M. Laukamp, G. B. Martins, C. Gazza, A. L. Malvezzi, E. Dagotto, P. M. Hansen, A. C. López, and J. Riera, Phys. Rev. B 57, 10755 (1998).
[2] M. Nishino, H. Onishi, K. Yamaguchi, and S. Miyashita, Phys. Rev. B 62, 9463 (2000).

[3] M. Nishino, H. Onishi, P. Roos, K. Yamaguchi, and S. Miyashita, Phys. Rev. B 61, 4033 (2000). 
[4] M. Takigawa, N. Motoyama, H. Eisaki, and S. Uchida, Phys. Rev. B 55, 14129 (1997).

[5] J. Sirker, N. Laflorencie, S. Fujimoto, S. Eggert, and I. Affleck, Phys. Rev. Lett. 98, 137205 (2007).

[6] K. M. Kojima, J. Yamanobe, H. Eisaki, S. Uchida, Y. Fudamoto, I. M. Gat, M. I. Larkin, A. Savici, Y. J. Uemura, P. P. Kyriakou, M. T. Rovers, and G. M. Luke, Phys. Rev. B 70, 094402 (2004).

[7] S. Bertaina, C.-E. Dutoit, J. Van Tol, M. Dressel, B. Barbara, and A. Stepanov, Phys. Rev. B 90, 060404 (2014).

[8] R. Kubo and K. Tomita, J. Phys. Soc. Jpn. 9, 888 (1954).

[9] R. Kubo, J. Phys. Soc. Jpn. 12, 570 (1957).

[10] K. Nagata and Y. Tazuke, J. Phys. Soc. Jpn. 32, 337 (1972).

[11] S. Miyashita, T. Yoshino, and A. Ogasahara, J. Phys. Soc. Jpn. 68, 655 (1999).

[12] A. Ogasahara and S. Miyshita, J. Phys. Soc. Jpn. 69, 4043 (2000).

[13] S. Miyashita and A. Ogasahara, J. Phys. Soc. Jpn. 72, 2350 (2003).

[14] S. El Shawish, O. Cépas, and S. Miyashita, Phys. Rev. B 81, 224421 (2010).

[15] M. Oshikawa and I. Affleck, Phys. Rev. Lett. 82, 5136 (1999); Phys. Rev. B 65, 134410 (2002).

[16] P. de Vries and H. De Raedt, Phys. Rev. B 47, 7929 (1993).

[17] T. Iitaka and T. Ebisuzaki, Phys. Rev. Lett. 90, 047203 (2003).

[18] M. Machida, T. Iitaka, and S. Miyashita, Phys. Rev. B 86, 224412 (2012).

[19] H. Tal-Ezer and R. Kosloff, J. Chem. Phys. 81, 3967 (1984).
[20] C. Leforestier, R. Bisseling, C. Cerjan, M. D. Feit, R. Friesner, A. Guldberg, A. Hammerich, G. Jolicard, W. Karrlein, H.-D. Meyer, N. Lipkin, O. Roncero, and R. Kosloff, J. Comp. Phys. 94, 59 (1991).

[21] V. V. Dobrovitski and H. A. De Raedt, Phys. Rev. E 67, 056702 (2003).

[22] T. Iitaka, S. Nomura, H. Hirayama, X. Zhao, Y. Aoyagi, and T. Sugano, Phys. Rev. E 56, 1222 (1997).

[23] A. Hams and H. De Raedt, Phys. Rev. E 62, 4365 (2000).

[24] H. De Raedt, A. H. Hams, K. Michielsen, S. Miyashita, and K. Saito, Prog. Theor. Phys. 138, 489 (2000).

[25] S. Sugiura and A. Shimizu, Phys. Rev. Lett. 108, 240401 (2012); 111, 010401 (2013).

[26] F. Jin, K. Michielsen, M. A. Novotny, S. Miyashita, S. Yuan, and H. De Raedt, Phys. Rev. A 87, 022117 (2013).

[27] R. Steinigeweg, F. Heidrich-Meisner, J. Gemmer, K. Michielsen, and H. De Raedt, Phys. Rev. B 90, 094417 (2014)

[28] See Supplemental Material at http://link.aps.org/supplemental/ 10.1103/PhysRevB.92.214431 for statistical properties of the thermal typical state approach.

[29] See Supplemental Material at http://link.aps.org/supplemental/ 10.1103/PhysRevB.92.214431 for conservation of intensity.

[30] F. J. Harris, Proc. IEEE 66, 51 (1978).

[31] N. G. Van Kampen, Stochastic Processes in Physics and Chemistry, 3rd ed. (Elsevier, Amsterdam, 2007).

[32] The relation to real measurements is a very interesting problem which will be discussed separately. 